\title{
Semi-Supervised Hashing for Large-Scale Search
}

\author{
Jun Wang, Member, IEEE, Sanjiv Kumar, Member, IEEE, and Shih-Fu Chang, Fellow, IEEE
}

\begin{abstract}
Hashing-based approximate nearest neighbor (ANN) search in huge databases has become popular due to its computational and memory efficiency. The popular hashing methods, e.g., Locality Sensitive Hashing and Spectral Hashing, construct hash functions based on random or principal projections. The resulting hashes are either not very accurate or are inefficient. Moreover, these methods are designed for a given metric similarity. On the contrary, semantic similarity is usually given in terms of pairwise labels of samples. There exist supervised hashing methods that can handle such semantic similarity, but they are prone to overfitting when labeled data are small or noisy. In this work, we propose a semi-supervised hashing (SSH) framework that minimizes empirical error over the labeled set and an information theoretic regularizer over both labeled and unlabeled sets. Based on this framework, we present three different semi-supervised hashing methods, including orthogonal hashing, nonorthogonal hashing, and sequential hashing. Particularly, the sequential hashing method generates robust codes in which each hash function is designed to correct the errors made by the previous ones. We further show that the sequential learning paradigm can be extended to unsupervised domains where no labeled pairs are available. Extensive experiments on four large datasets (up to 80 million samples) demonstrate the superior performance of the proposed SSH methods over state-of-the-art supervised and unsupervised hashing techniques.
\end{abstract}

Index Terms-Hashing, nearest neighbor search, binary codes, semi-supervised hashing, pairwise labels, sequential hashing

\section{INTRODUCTION}

W EB data including documents, images, and videos are growing rapidly. For example, the photo sharing website Flickr has over 5 billion images. The video sharing website YouTube receives more than 24 hours of uploaded videos per minute. There is an emerging need to retrieve relevant content from such massive databases. Besides the widely used text-based commercial search engines like Google and Bing, content-based image retrieval (CBIR) has attracted substantial attention over the past decade [1]. Instead of taking textual keywords as input, CBIR techniques directly take a visual query $q$ and try to return its nearest neighbors from a given database $\mathcal{X}$ using a prespecified feature space and distance measure.

In fact, finding nearest neighbors is a fundamental step in many machine learning algorithms such as kernel density estimation, spectral clustering, manifold learning, and semi-supervised learning [2]. Exhaustively comparing the query $q$ with each sample in the database $\mathcal{X}$ is infeasible because linear complexity $\mathcal{O}(|\mathcal{X}|)$ is not scalable in practical settings. Besides the scalability issue, most large-scale CBIR applications also suffer from the curse of dimensionality since

- J. Wang is with the Business Analytics and Mathematical Sciences Department, IBM T.J. Watson Research Center, RM 31-229, 1101 Kitchawan Rd, Rte. 134, Yorktown Heights, NY 10598.

E-mail:wangjun@us.ibm.com.

- S. Kumar is with Google Research, 76 Ninth Avenue, 4th Floor, New York, NY 10011.E-mail: sanjivk@google.com.

- S.-F. Chang is with the Department of Electrical and Computer Engineering, Columbia University, RM 1312, 500 W. 120th St., New York, NY 10027. E-mail: sfchang@ee.columbia.edu.

Manuscript received 15 Dec. 2010; revised 1 Aug. 2011; accepted 28 Jan. 2012; published online 6 Feb. 2012.

Recommended for acceptance by C. Stewart.

For information on obtaining reprints of this article, please send e-mail to: tpami@computer.org, and reference IEEECS Log Number

TPAMI-2010-12-0959.

Digital Object Identifier no. 10.1109/TPAMI.2012.48. visual descriptors usually have hundreds, or even thousands, of dimensions. Therefore, beyond the infeasibility of exhaustive search, storage of the original data also becomes a critical bottleneck.

Fortunately, in many applications it is sufficient to return approximate nearest neighbors (ANN). Instead of doing an exact nearest neighbor search through linear scan, a fast and accurate indexing method with sublinear $(o(|\mathcal{X}|))$, logarith$\operatorname{mic}(\mathcal{O}(\log |\mathcal{X}|))$, or even constant $(\mathcal{O}(1))$ query time is desired for approximate nearest neighbors search. For example, $\epsilon$ ANN aims at finding $p \in \mathcal{X}$ satisfying $d(p, q) \leq(1+\epsilon) d\left(p^{\prime}, q\right)$, where $\epsilon>0$ and $p^{\prime}$ is the nearest neighbor of query $q$ [3]. Over the past several decades, many techniques have been developed for fast and efficient ANN search. Especially, tree-based approaches tend to store data with efficient data structures, which makes the search operation extremely fast, typically with the complexity of $\mathcal{O}(\log (|\mathcal{X}|))$. The representative tree-based algorithms include KD tree [4], [5], [6], ball tree [7], metric tree [8], and vantage point tree [9]. A more detailed survey of the tree-based ANN search algorithms can be found in [10]. However, the performance of tree-based methods is drastically degraded for high-dimensional data, being mostly reduced to the worst case of a linear search [11].

In addition, tree-based methods also suffer from memory constraints. In many cases, the size of the data structure is bigger than the original data itself. Hence, hashing-based ANN techniques have attracted more attention recently. They have constant query time and also need substantially reduced storage as they usually store only compact codes. In this work, we focus on binary codes. Given $n D$-dim vectors $\mathbf{X} \in \mathbb{R}^{D \times n}$, the goal in hashing is to learn suitable $K$-bit binary codes $\mathbf{Y} \in \mathbb{B}^{K \times n}$. To generate $\mathbf{Y}, K$ binary hash functions are used. Linear projection-based hash functions have been widely used in the literature since they are very simple and efficient. Also, they have achieved 
state-of-the-art performance for various tasks [12], [13], [14]. In linear projection-based hashing, the $k$ th hash function can be generalized to be of the following form:

$$
h_{k}(\mathbf{x})=\operatorname{sgn}\left(f\left(\mathbf{w}_{k}^{\top} \mathbf{x}+b_{k}\right)\right)
$$

where $\mathbf{x}$ is a data point, $\mathbf{w}_{k}$ is a projection vector, $b_{k}$ is a threshold, and $f(\cdot)$ is an arbitrary function. Since $h(\mathbf{x}) \in\{-1,1\}$, the corresponding binary hash bit can be simply expressed as $y_{k}(\mathbf{x})=\left(1+h_{k}(\mathbf{x})\right) / 2$. Different choices of $\mathbf{w}$ and $f(\cdot)$ lead to different hashing approaches.

Broadly, hashing methods can be divided into two main categories: unsupervised methods and supervised methods. Unsupervised methods design hash functions using unlabeled data $\mathcal{X}$ to generate binary codes. Locality Sensitive Hashing $(L S H)$ [12] is arguably the most popular unsupervised hashing method and has been applied to many problem domains, including information retrieval and computer vision. Its kernelized version has recently been developed in [15]. Another effective method, called Spectral Hashing $(\mathrm{SH})$, was proposed recently by Weiss et al. [13]. More recently, graph based hashing technique was proposed to leverage low-dimensional manifold structure of data to design efficient and compact hash codes [39]. Since unsupervised methods do not require any labeled data, they can be easily applied to different data domains given a prespecified distance metric.

From the perspective of quality of retrieved results, hashing-based ANN methods aim to return an approximate set of nearest neighbors. However, in typical CBIR, even returning the exact nearest neighbors does not guarantee good search quality. This is due to the well-known problem of semantic gap, where the high-level semantic description of visual content often differs from the extracted low-level visual descriptors [16]. Furthermore, most hashing approaches provide theoretic guarantees with only certain distance metric spaces. For instance, the $L S H$ function family works for the $\ell_{p}(p \in(0,2])$ and Jaccard distances. But in CBIR applications, it is usually hard to express similarity (or distance) between image pairs with a simple metric. Ideally, one would like to provide pairs of images that one believes contain "similar" or "dissimilar" images. From such pairwise labeled data, the hashing mechanism should be able to automatically generate codes that satisfy the semantic similarity. Supervised learning techniques have been proposed in the past to handle this issue. For example, in [17], the authors suggested merging standard $L S H$ with a learned Mahalanobis metric to reflect semantic indexing. Since this approach uses labeled sample pairs for training distance metric, it was categorized as a semisupervised learning paradigm. However, the hash functions are still randomized.

In addition, a Boosted Similarity Sensitive Coding (BSSC) technique was proposed in [18] which tries to learn a series of weighted hash functions from labeled data. Kulis and Darrell recently proposed learning hash functions based on explicitly minimizing the reconstruction error between the metric space and Hamming space, termed Binary Reconstructive Embedding $(B R E)$ [20]. Realizing the difficulty of optimizing Hamming distance-based objective functions, Liu et al. explored the equivalence between the code inner products and the Hamming distances to design efficient kernelized hash functions with pairwise labels [40]. Other binary encoding methods, like deep neural network stacked with Restricted Boltzmann Machines ( $R B M s)$, were recently applied to learn binary codes [19], which have shown superior performance over BSSC given sufficient training labels [22]. Although RBMs use both labeled and unlabeled data, the latter is only used in a pretraining phase, whose solution provides a good initialization for the supervised back-propagation phase. So, RBMs-based binary embedding is still categorized as a supervised method. One of the problems with all of these supervised methods is that they have a much slower training process in comparison to the unsupervised methods. Another problem stems from limited or noisy training data, which can easily lead to overfitting.

Realizing the challenging issue of semantic gap and the inefficiencies of existing supervised hashing approaches, in this paper we propose a Semi-Supervised Hashing (SSH) framework that can leverage semantic similarity using labeled data while remaining robust to overfitting. The objective function of $S S H$ consists of two main components: supervised empirical fitness and unsupervised information theoretic regularization. Specifically, we provide a rigorous formulation in which a supervised term tries to minimize the empirical error on the labeled data while an unsupervised term provides effective regularization by maximizing desirable properties like variance and independence of individual bits. Based on this semi-supervised formulation, we present several variants of learning binary hash functions. The taxonomy of the proposed semi-supervised hashing method in comparison to a few popular hashing methods is given in Table 1.

The remainder of this paper is organized as follows: In Section 2, we briefly survey several popular hashing methods. Section 3 presents the detailed formulation of our approach, i.e., Semi-Supervised Hashing. In Section 4, we present three different solutions for designing semisupervised hash functions, followed by an extension to unsupervised domain. Section 5 provides extensive experimental validation on several large image datasets. The conclusions and future work are given in Section 6.

\section{Related Work}

Given a dataset $\mathbf{X}=\left\{\mathbf{x}_{i}\right\}, i=1, \ldots, n$, containing $n=|\mathbf{X}|$ points, and $\mathbf{x}_{i} \in \mathbb{R}^{D}$, the objective in nearest neighbor search is to find a set of nearest neighbors $\mathcal{R} \subset \mathbf{X}$ for a given query $q$. For large-scale applications, to avoid excessive computational and memory costs one would like to instead do an ANN search with sublinear query complexity [2], [12].

In the following sections, in addition to the popularly used Locality Sensitive Hashing, we briefly review a few state-ofthe-art representative methods from supervised as well as unsupervised domains. Specifically, we discuss Boosted Similarity Sensitive Coding, Spectral Hashing, and Binary Reconstructive Embedding-based hashing along with their pros and cons for the application of image retrieval.

\subsection{Locality Sensitive Hashing}

A key ingredient of Locality Sensitive Hashing is mapping "similar" samples to the same bucket with high probability. In other words, the property of locality in the original space 
TABLE 1

The Conceptual Comparison of the Proposed SSH Method with Other Binary Encoding Methods

\begin{tabular}{|c|c|c|c|c|}
\hline Method & Hash Function & Projection & Hamming Distance & Learning Paradigm \\
\hline \hline Locality Sensitive Hashing (LSH) [12] & $\operatorname{sgn}\left(\mathbf{w}^{\top} \mathbf{x}+b\right)$ & data-independent & non-weighted & unsupervised \\
\hline Shift Invariant Kernel based Hashing (SIKH) [14] & $\operatorname{sgn}\left(\cos \left(\mathbf{w}^{\top} \mathbf{x}+b\right)+t\right)$ & data-independent & non-weighted & unsupervised \\
\hline Spectral Hashing (SH) [13] & $\operatorname{sgn}\left(\cos \left(k \mathbf{w}^{\top} \mathbf{x}\right)\right)$ & data-dependent & non-weighted & unsupervised \\
\hline Boosted Similarity Sensitive Coding (BSSC) [18] & - & data-dependent & weighted & supervised \\
\hline Restricted Boltzmann Machines (RBMs) [19] & - & - & non-weighted & supervised \\
\hline Binary Reconstructive Embedding (BRE) [20] & $\operatorname{sgn}\left(\mathbf{w}^{\top} \mathbf{k}_{\mathbf{x}}\right)$ & data-dependent & non-weighted & supervised \\
\hline Label-regularized Max-margin Partition (LAMP) [21] & $\operatorname{sgn}\left(\mathbf{w}^{\top} \mathbf{x}+b\right)$ & data-dependent & non-weighted & supervised \\
\hline LSH for Learned Metrics (LSH-LM) [17] & $\operatorname{sgn}\left(\mathbf{w}^{\top} \mathbf{G} \mathbf{x}\right)$ & data-dependent & non-weighted & supervised \\
\hline Semi-Supervised Hashing (SSH) & $\operatorname{sgn}\left(\mathbf{w}^{\top} \mathbf{x}+b\right)$ & data-dependent & non-weighted & semi-supervised \\
\hline
\end{tabular}

will be largely preserved in the Hamming space. More precisely, the hash functions $h(\cdot)$ from the LSH family satisfy the following elegant locality preserving property:

$$
P\{h(\mathbf{x})=h(\mathbf{y})\}=\operatorname{sim}(\mathbf{x}, \mathbf{y}),
$$

where the similarity measure can be directly linked to the distance function $d$, for example, $\operatorname{sim}(\mathbf{x}, \mathbf{y})=\exp \left\{-\frac{\|\mathbf{x}-\mathbf{y}\|^{2}}{\sigma^{2}}\right\}$. A typical category of $L S H$ functions consists of random projections and thresholds as:

$$
h(x)=\operatorname{sign}\left(\mathbf{w}^{\top} \mathbf{x}+b\right),
$$

where $\mathbf{w}$ is a random hyperplane and $b$ is a random intercept. Clearly, the random vector $\mathbf{w}$ is data independent, which is usually constructed by sampling each component of $\mathbf{w}$ randomly from a $p$-stable distribution for a general $\ell_{p}$ metric, where $p \in(0,2]$, e.g., standard Gaussian for $\ell_{2}$ distance [23]. Due to the asymptotic theoretical guarantees for random projection-based $L S H$, many $L S H$ based large-scale search systems have been developed. For instance, a self-tuning indexing technique, called LSH forest, was proposed in [24], which aims at improving the performance without additional storage and query overhead. However, the practical efficiency of $L S H$ is still very limited since it requires long codes to achieve high precision. But this reduces recall dramatically and constructing multiple tables is necessary to increase recall [12]. For example, suppose a K-bit binary embedding is given as $H(x)=\left[h_{1}(x), \ldots, h_{K}(x)\right]$. Then, given $l K$-bit tables, the collision probability for two points is given as

$$
P\{H(\mathbf{x})=H(\mathbf{y})\} \propto l \cdot\left[1-\frac{\cos ^{-1} \mathbf{x}^{\top} \mathbf{y}}{\pi}\right]^{K} .
$$

For a large-scale application, the value of $K$ should be large to reduce false collisions (i.e., the number of nonneighbor sample pairs falling into the same bucket). However, a large value of $K$ decreases the collision probability between similar samples as well. In order to overcome this drawback, multiple hash tables have to be constructed. Obviously, this is inefficient due to extra storage cost and larger query time. In [25], a technique called MultiProbe $L S H$ was developed to reduce the number of required hash tables through intelligently probing multiple buckets in each hash table. However, the above data-independent random projections-based hash functions lack good discrimination over data. Therefore, recent methods tend to leverage data-dependent learning techniques to improve the efficiency of hash functions [26].

Incorporating kernel learning with LSH can help generalize similarity search from standard metric space to a wide class of similarity functions [15], [21]. Furthermore, metric learning has been combined with randomized $L S H$ functions given a few pairwise similarity and dissimilarity constraints [17]. All these methods still use random hyperplanes to design hash functions with asymptotic performance guarantees. However, in practice, the performance is typically poor if only compact codes are used [13], [27].

\subsection{Boosted Similarity Sensitive Coding}

To improve discrimination among hash codes, Boosted Similarity Sensitive Coding was designed to learn a weighted Hamming embedding for task specific similarity search [18] as

$$
H: \mathcal{X} \rightarrow\left\{\alpha_{1} h_{1}(\mathbf{x}), \ldots, \alpha_{K} h_{K}(\mathbf{x})\right\} .
$$

Hence, the conventional Hamming distance is replaced by the weighted version as

$$
d_{\mathcal{W H}}=\sum_{k=1}^{K} \alpha_{k}\left|h_{k}\left(\mathbf{x}_{i}\right)-h_{k}\left(\mathbf{x}_{j}\right)\right| .
$$

By learning the hash function weights $\left\{\alpha_{1}, \ldots, \alpha_{k}\right\}$, the objective is to lower the collision probability of nonneighbor pair $\left(\mathbf{x}_{i}, \mathbf{x}_{j}\right) \in \mathcal{C}$ while improving the collision probability of neighborhood pair $\left(\mathbf{x}_{i}, \mathbf{x}_{j}\right) \in \mathcal{M}$. If one treats each hash function as a decision stump, the straightforward way of learning the weights is to directly apply adaptive boosting algorithm [28], as described in [18].

\subsection{Spectral Hashing}

Due to the limitations of random projection-based hashing approaches, learning techniques have been applied to improve the efficiency of hashing. Particularly, Spectral Hashing was recently proposed to design compact binary codes for ANN search. Besides the desired property of keeping neighbors in input space as neighbors in the Hamming space, the basic $S H$ formulation requires the codes to be balanced and uncorrelated. Strictly speaking, the hash 
functions $H(\mathbf{x})=\left\{h_{k}(\mathbf{x})\right\}, k=1, \ldots, K$ satisfy the following criteria [13]:

$$
\begin{aligned}
\min & \sum_{i j} \operatorname{sim}\left(\mathbf{x}_{i}, \mathbf{x}_{j}\right)\left\|H\left(\mathbf{x}_{i}\right)-H\left(\mathbf{x}_{j}\right)\right\|^{2} \\
\text { subject to: } & h_{k}\left(\mathbf{x}_{i}\right) \in\{-1,1\} \\
& \sum_{i} h_{k}\left(\mathbf{x}_{i}\right)=0, \quad k=1, \ldots, K \\
& \sum_{i} h_{k}\left(\mathbf{x}_{i}\right) h_{l}\left(\mathbf{x}_{i}\right)=0, \quad \text { for } k \neq l .
\end{aligned}
$$

The direct solution for the above optimization is nontrivial for even a single bit since it is a balanced graph partition problem, which is NP-hard. The combination of $K$-bit balanced partitioning is even harder because of the pairwise independence constraints. After relaxing the constraints, the above optimization was solved using spectral graph analysis [29]. Especially with the assumption of uniform data distribution, the spectral solution can be efficiently computed using 1D-Laplacian eigenfunctions [13].

The final $\mathrm{SH}$ algorithm consists of three key steps: 1) extraction of maximum variance directions through Principal Component Analysis (PCA) on the data, 2) direction selection, which prefers to partition projections with large spread and small spatial frequency, 3) partition of projected data by a sinusoidal function with previously computed angular frequency. SH has been shown to be effective in encoding low-dimensional data since the important PCA directions are selected multiple times to create binary bits. However, for high-dimensional problems $(D \gg K)$ where many directions contain enough variance, usually each PCA direction is picked only once. This is because the top few projections have similar range and thus a low spatial frequency is preferred. In this case, SH approximately replicates a PCA projection followed by a mean partition. In $\mathrm{SH}$, the projection directions are data dependent but learned in an unsupervised manner. Moreover, the assumption of uniform data distribution is usually not true for real-world data.

\subsection{Binary Reconstructive Embedding}

Instead of using data-independent random projections as in $L S H$ or principal components as in $S H$, Kulis and Darrell [20] proposed data-dependent and bit-correlated hash functions as

$$
h_{k}(\mathbf{x})=\operatorname{sgn}\left(\sum_{q=1}^{s} \mathbf{W}_{k q} \kappa\left(\mathbf{x}_{k q}, \mathbf{x}\right)\right) .
$$

The sample set $\left\{\mathbf{x}_{k q}\right\}, q=1, \ldots, s$, is the training data for learning hash function $h_{k}$ and $\kappa(\cdot)$ is a kernel function and $\mathrm{W}$ is a weight matrix.

Based on the above formulation, a method called Binary Reconstructive Embedding was designed to minimize a cost function measuring the difference between the metric and reconstructed distance in Hamming space. The euclidean metric $d_{\mathcal{M}}$ and the binary reconstruction distance $d_{\mathcal{R}}$ are defined as

$$
\begin{aligned}
& d_{\mathcal{M}}\left(\mathbf{x}_{i}, \mathbf{x}_{j}\right)=\frac{1}{2}\left\|\mathbf{x}_{i}-\mathbf{x}_{j}\right\|^{2} \\
& d_{\mathcal{R}}\left(\mathbf{x}_{i}, \mathbf{x}_{j}\right)=\frac{1}{K} \sum_{k=1}^{K}\left(h_{k}\left(\mathbf{x}_{i}\right)-h_{k}\left(\mathbf{x}_{j}\right)\right)^{2}
\end{aligned}
$$

The objective is to minimize the following reconstruction error to derive the optimal $\mathbf{W}$ :

$$
\mathbf{W}^{*}=\arg \min _{\mathbf{W}} \sum_{\left(\mathbf{x}_{i}, \mathbf{x}_{j}\right) \in \mathcal{N}}\left[d_{\mathcal{M}}\left(\mathbf{x}_{i}, \mathbf{x}_{j}\right)-d_{\mathcal{R}}\left(\mathbf{x}_{i}, \mathbf{x}_{j}\right)\right]^{2},
$$

where the set of sample pairs $\mathcal{N}$ is the training data. Optimizing the above objective function is difficult due to the nondifferentiability of $\operatorname{sgn}(\cdot)$ function. Instead, a coordinate-descent algorithm was applied to iteratively update the hash functions to a local optimum. This hashing method can be easily extended to a supervised scenario by setting the same-label pairs to have zero distance and different-label pairs to have a large distance. However, since the binary reconstruction distance $d_{\mathcal{R}}$ is bounded in $[0,1]$ while the metric distance $d_{\mathcal{M}}$ has no upper bound, the minimization problem in (10) is only meaningful when input data are appropriately normalized. In practice, the original data point $\mathrm{x}$ is often mapped to a hypersphere with unit length so that $0 \leq d_{\mathcal{M}} \leq 1$. This normalization removes the scale of data points, which is often not negligible in practical applications of nearest neighbor search.

\section{Semi-Supervised Paradigm for Hashing}

In this section, we present the formulation of our hashing method, i.e., Semi-Supervised Hashing. In this setting, one is given a set of $n$ points, $\mathcal{X}=\left\{\mathbf{x}_{i}\right\}, i=1 \ldots n, \mathbf{x}_{i} \in \mathbb{R}^{D}$, in which a fraction of pairs are associated with two categories of label information, $\mathcal{M}$ and $\mathcal{C}$. Specifically, a pair $\left(\mathbf{x}_{i}, \mathbf{x}_{j}\right) \in$ $\mathcal{M}$ is denoted as a neighbor pair when $\left(\mathbf{x}_{i}, \mathbf{x}_{j}\right)$ are neighbors in a metric space or share common class labels. Similarly, $\left(\mathbf{x}_{i}, \mathbf{x}_{j}\right) \in \mathcal{C}$ is called a nonneighbor pair if two samples are far away in metric space or have different class labels. Let us denote the data matrix by $\mathbf{X} \in \mathbb{R}^{D \times n}$, where each column is a data point. Also, suppose there are $l$ points, $l \ll n$, which are associated with at least one of the categories $\mathcal{M}$ or $\mathcal{C}$. Let us denote the matrix formed by these $l$ columns of $\mathbf{X}$ as $\mathbf{X}_{l} \in \mathbb{R}^{D \times l}$. The goal of $S S H$ is to learn hash functions that minimize the error on the labeled training data $\mathbf{X}_{l}$ while maximally satisfying the desirable properties of hashing, e.g., maximizing information from each bit. We start the discussion on our learning paradigm with the basic formulation of $\mathrm{SSH}$.

\subsection{Empirical Fitness}

SSH aims to map the data $\mathbf{X} \in \mathbb{R}^{D \times n}$ to a Hamming space to obtain its compact representation. Suppose we want to learn $K$ hash functions leading to a $K$-bit Hamming embedding of $\mathbf{X}$ given by $\mathbf{Y} \in \mathbb{B}^{K \times n}$. Without loss of generality, let $\mathbf{X}$ be normalized to have zero mean. In this work, we use linear projection coupled with mean thresholding as a hash function. In other words, given a vector $\mathbf{w}_{k} \in \mathbb{R}^{D}$, the $k$ th hash function is defined as

$$
h_{k}\left(\mathbf{x}_{i}\right)=\operatorname{sgn}\left(\mathbf{w}_{k}^{\top} \mathbf{x}_{i}+b_{k}\right),
$$

where $b_{k}$ is the mean of the projected data, i.e., $b_{k}=$ $-\frac{1}{n} \sum_{j=1}^{n} \mathbf{w}_{k}^{\top} \mathbf{x}_{j}=0$ since $\mathbf{X}$ is zero mean. One can get the corresponding binary bit as

$$
y_{k i}=\frac{1}{2}\left(1+h_{k}\left(\mathbf{x}_{i}\right)\right)=\frac{1}{2}\left(1+\operatorname{sgn}\left(\mathbf{w}_{k}^{\top} \mathbf{x}_{i}\right)\right) .
$$


Let $\mathbf{H}=\left[h_{1}, \ldots, h_{K}\right]$ be a sequence of $K$ hash functions and $\mathbf{W}=\left[\mathbf{w}_{1}, \ldots, \mathbf{w}_{K}\right] \in \mathbb{R}^{D \times K}$. We want to learn a $\mathbf{W}$ that gives the same bits for $\left(\mathbf{x}_{i}, \mathbf{x}_{j}\right) \in \mathcal{M}$ and different bits for $\left(\mathbf{x}_{i}, \mathbf{x}_{j}\right) \in \mathcal{C}$. An objective function measuring the empirical accuracy on the labeled data for a family of hashing functions $\mathbf{H}$ can be defined as

$$
J(\mathbf{H})=\sum_{k}\left\{\sum_{\left(\mathbf{x}_{i}, \mathbf{x}_{j}\right) \in \mathcal{M}} h_{k}\left(\mathbf{x}_{i}\right) h_{k}\left(\mathbf{x}_{j}\right)-\sum_{\left(\mathbf{x}_{i}, \mathbf{x}_{j}\right) \in \mathcal{C}} h_{k}\left(\mathbf{x}_{i}\right) h_{k}\left(\mathbf{x}_{j}\right)\right\} .
$$

One can express the above objective function in a compact matrix form by first defining a matrix $\mathbf{S} \in \mathbb{R}^{l \times l}$ incorporating the pairwise labeled information from $\mathbf{X}_{l}$ as

$$
S_{i j}=\left\{\begin{aligned}
1: & \left(\mathbf{x}_{i}, \mathbf{x}_{j}\right) \in \mathcal{M} \\
-1: & \left(\mathbf{x}_{i}, \mathbf{x}_{j}\right) \in \mathcal{C} \\
0: & \text { otherwise }
\end{aligned}\right.
$$

Also, suppose $\mathbf{H}\left(\mathbf{X}_{l}\right) \in \mathbb{B}^{K \times l}$ maps the points in $\mathbf{X}_{l}$ to their K-bit hash codes. Then, the objective function $J(\mathbf{H})$ can be represented as

$$
\begin{aligned}
J(\mathbf{H}) & =\frac{1}{2} \operatorname{tr}\left\{\mathbf{H}\left(\mathbf{X}_{l}\right) \mathbf{S H}\left(\mathbf{X}_{l}\right)^{\top}\right\} \\
& =\frac{1}{2} \operatorname{tr}\left\{\operatorname{sgn}\left(\mathbf{W}^{\top} \mathbf{X}_{l}\right) \mathbf{S} \operatorname{sgn}\left(\mathbf{W}^{\top} \mathbf{X}_{l}\right)^{\top}\right\}
\end{aligned}
$$

where $\operatorname{sgn}\left(\mathbf{W}^{\top} \mathbf{X}_{l}\right)$ is the matrix of signs of individual elements. In summary, we intend to learn optimal hashing functions $\mathbf{H}$ by maximizing the objective function as

$$
\mathbf{H}^{*}=\arg \max _{\mathbf{H}} J(\mathbf{H}) .
$$

Since the objective function $J(\mathbf{H})$ itself is nondifferentiable, the above problem is difficult to solve even without considering any regularizer. We first present a simple relaxation of the empirical fitness.

In the relaxed version of the objective function, we replace the sign of projection with its signed magnitude in (13). This relaxation is quite intuitive in the sense that it not only desires similar points to have the same sign but also large projection magnitudes, meanwhile projecting dissimilar points not only with different signs but also as far as possible. With this relaxation, the new objective can be directly written as a function of $\mathbf{W}$ as

$$
J(\mathbf{W})=\sum_{k}\left\{\sum_{\left(\mathbf{x}_{i}, \mathbf{x}_{j}\right) \in \mathcal{M}} \mathbf{w}_{k}^{\top} \mathbf{x}_{i} \mathbf{x}_{j}^{\top} \mathbf{w}_{k}-\sum_{\left(\mathbf{x}_{i}, \mathbf{x}_{j}\right) \in \mathcal{C}} \mathbf{w}_{k}^{\top} \mathbf{x}_{i} \mathbf{x}_{j}^{\top} \mathbf{w}_{k}\right\}
$$

Without loss of generality, we also assume $\left\|\mathbf{w}_{k}\right\|=1, \forall k$. The above function can be expressed in a matrix form as

$$
J(\mathbf{W})=\frac{1}{2} \operatorname{tr}\left\{\mathbf{W}^{\top} \mathbf{X}_{l} \mathbf{S} \mathbf{X}_{l}^{\top} \mathbf{W}\right\} .
$$

\subsection{Information Theoretic Regularization}

Maximizing empirical accuracy for just a few pairs can lead to severe overfitting, as illustrated in Fig. 1. To get better generalization ability, one needs to add regularization by incorporating conditions that lead to desirable properties of
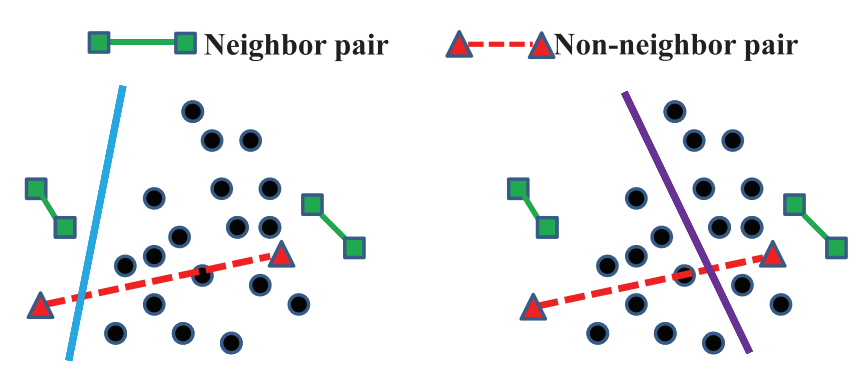

Fig. 1. An illustration of partitioning with maximum empirical fitness and entropy. Both of the partitions satisfy the given pairwise labels, while the right one is more informative due to higher entropy.

hash codes. Even though empirical fitness uses only labeled data, the regularization term uses all the data $X$, both unlabeled and labeled, leading to a semi-supervised learning paradigm. Hence, we use a regularizer which utilizes both labeled and unlabeled data. From the information-theoretic point of view, one would like to maximize the information provided by each bit [30]. Using maximum entropy principle, a binary bit that gives balanced partitioning of $\mathbf{X}$ provides maximum information. Thus, it is desired to have $\sum_{i=1}^{n} h_{k}\left(\mathbf{x}_{i}\right)=0$. However, finding mean-thresholded hash functions that meet the balancing requirement is hard. Instead, we use this property to construct a regularizer for the empirical accuracy given in (18). We now show that maximum entropy partitioning is equivalent to maximizing the variance of a bit.

Proposition 3.1 (Maximum variance condition). A hash function with maximum entropy $H\left(h_{k}(\mathbf{x})\right)$ must maximize the variance of the hash values and vice versa, i.e.,

$$
\max H\left(h_{k}(\mathbf{x})\right) \Longleftrightarrow \max \operatorname{var}[h(\mathbf{x})] \text {. }
$$

Proof. Assume $h_{k}$ has a probability $p$ of assigning the hash value $h_{k}(\mathbf{x})=1$ to a data point and $1-p$ for $h_{k}(\mathbf{x})=-1$. The entropy of $h_{k}(\mathbf{x})$ can be computed as

$$
H\left(h_{k}(\mathbf{x})\right)=-p \log _{2} p-(1-p) \log _{2}(1-p) .
$$

It is easy to show that the maximum entropy is $\max H\left(h_{k}(\mathbf{x})\right)=\mathbf{1}$ when the partition is balanced, i.e., $p=1 / 2$. Now, we show that balanced partitioning implies maximum bit variance. The mean of hash value is $E[h(\mathbf{x})]=\mu=2 p-1$ and the variance is

$$
\begin{aligned}
& \operatorname{var}\left[h_{k}(\mathbf{x})\right]=E\left[\left(h_{k}(\mathbf{x})-\mu\right)^{2}\right] \\
& \quad=4(1-p)^{2} p+4 p^{2}(1-p)=4 p(1-p) .
\end{aligned}
$$

Clearly, $\operatorname{var}[h(\mathbf{x})]$ is concave with respect to $p$ and its maximum is reached at $p=1 / 2$, i.e., balanced partitioning. Also, since $\operatorname{var}[h(\mathbf{x})]$ has a unique maximum, it is easy to see that the maximum variance partitioning also maximizes the entropy of the hash function.

Using the above proposition, the regularizer term is defined as

$$
R(\mathbf{W})=\sum_{k} \operatorname{var}\left[h_{k}(\mathbf{x})\right]=\sum_{k} \operatorname{var}\left[\operatorname{sgn}\left(\mathbf{w}_{k}^{\top} \mathbf{x}\right)\right] .
$$

Maximizing the above function with respect to $\mathbf{W}$ is still hard due to its nondifferentiability. To overcome this problem, we now show that the maximum variance of a 
hash function is lower bounded by the scaled variance of the projected data.

Proposition 3.2 (Lower bound on maximum variance of a hash function). The maximum variance of a hash function is lower bounded by the scaled variance of the projected data, i.e.,

$$
\max \operatorname{var}\left[h_{k}(\mathbf{x})\right] \geq \alpha \cdot \operatorname{var}\left[\mathbf{w}_{k}^{\top} \mathbf{x}\right]
$$

where $\alpha$ is a positive constant.

Proof. Suppose $\left\|\mathbf{x}_{i}\right\|^{2} \leq \beta \forall i, \beta>0$. Since $\left\|\mathbf{w}_{k}\right\|^{2}=1 \forall k$, from the Cauchy-Schwarz inequality:

$$
\begin{gathered}
\left\|\mathbf{w}_{k}^{\top} \mathbf{x}\right\|^{2} \leq\left\|\mathbf{w}_{k}\right\|^{2} \cdot\|\mathbf{x}\|^{2} \leq \beta=\beta \cdot\left\|\operatorname{sgn}\left(\mathbf{w}_{k}^{\top} \mathbf{x}\right)\right\|^{2} \\
\Rightarrow E\left[\left\|\operatorname{sgn}\left(\mathbf{w}_{k}^{\top} \mathbf{x}\right)\right\|^{2}\right] \geq \frac{1}{\beta} E\left[\left\|\mathbf{w}_{k}^{\top} \mathbf{x}\right\|^{2}\right] \\
\Rightarrow \max \operatorname{var}\left[h_{k}(\mathbf{x})\right] \geq \frac{1}{\beta} \operatorname{var}\left[\mathbf{w}_{k}^{\top} \mathbf{x}\right] .
\end{gathered}
$$

Here, we have used the properties that the data are zero centered, i.e., $E\left[\mathbf{w}_{k}^{\top} \mathbf{x}\right]=0$, and for maximum bit variance $E\left[\operatorname{sgn}\left(\mathbf{w}_{k}^{\top} \mathbf{x}\right)\right]=0$.

Given the above proposition, we use the lower bound on the maximum variance of a hash function as a regularizer, which is easy to optimize, i.e.,

$$
\begin{aligned}
R(\mathbf{W}) & =\frac{1}{\beta} \sum_{k} E\left[\left\|\mathbf{w}_{k}^{\top} \mathbf{x}\right\|^{2}\right]=\frac{1}{n \beta} \sum_{k} \mathbf{w}_{k}^{\top} \mathbf{X} \mathbf{X}^{\top} \mathbf{w}_{k} \\
& =\frac{1}{n \beta} \operatorname{tr}\left[\mathbf{W}^{\top} \mathbf{X} \mathbf{X}^{\top} \mathbf{W}\right] .
\end{aligned}
$$

\subsection{Final Objective Function}

Combining the relaxed empirical fitness term from (18) and the relaxed regularization term from (20), the overall semisupervised objective function is given as

$$
\begin{aligned}
J(\mathbf{W}) & =\frac{1}{2} \operatorname{tr}\left[\mathbf{W}^{\top} \mathbf{X}_{l} \mathbf{S} \mathbf{X}_{l}^{\top} \mathbf{W}\right]+\frac{\eta}{2} \operatorname{tr}\left[\mathbf{W}^{\top} \mathbf{X} \mathbf{X}^{\top} \mathbf{W}\right] \\
& =\frac{1}{2} \operatorname{tr}\left\{\mathbf{W}^{\top}\left[\mathbf{X}_{l} \mathbf{S} \mathbf{X}_{l}^{\top}+\eta \mathbf{X} \mathbf{X}^{\top}\right] \mathbf{W}\right\} \\
& =\frac{1}{2} \operatorname{tr}\left\{\mathbf{W}^{\top} \mathbf{M W}\right\},
\end{aligned}
$$

where the constants $n$ and $\beta$ are absorbed in the coefficient $\eta$ and

$$
\mathbf{M}=\mathbf{X}_{l} \mathbf{S} \mathbf{X}_{l}^{\top}+\eta \mathbf{X} \mathbf{X}^{\top} .
$$

We refer to matrix $\mathbf{M}$ as the adjusted covariance matrix. It is interesting to note the form of $\mathbf{M}$, where the unsupervised data variance part $\mathbf{X X}^{T}$ gets adjusted by $\mathbf{X}_{l} \mathbf{S X}_{l}^{\top} \mathbf{W}$ arising from the pairwise labeled data.

\section{Projection Learning}

\subsection{Orthogonal Projection Learning}

While learning compact codes, in addition to each bit being highly informative, one would like to avoid redundancy in bits as much as possible. One way to achieve this is by making the projection directions orthogonal, i.e.,

$$
\begin{aligned}
\mathbf{W}^{*} & =\arg \max _{\mathbf{W}} J(\mathbf{W}) \\
\text { subject to } & \mathbf{W}^{\top} \mathbf{W}=\mathbf{I} .
\end{aligned}
$$

Now, the learning of optimal projections $\mathbf{W}$ becomes a typical eigenproblem, which can be easily solved by doing an eigenvalue decomposition on matrix $\mathbf{M}$ :

$$
\begin{aligned}
& \max _{\mathbf{W}} J(\mathbf{W})=\sum_{k=1}^{K} \lambda_{k} \\
& \mathbf{W}^{*}=\left[\mathbf{e}_{1} \cdots \mathbf{e}_{K}\right],
\end{aligned}
$$

where $\lambda_{1}>\lambda_{2}>\cdots>\lambda_{K}$ are the top eigenvalues of $\mathbf{M}$ and $\mathbf{e}_{k}, k=1, \ldots, K$, are the corresponding eigenvectors.

Mathematically, it is very similar to finding maximum variance direction using PCA except that the original covariance matrix gets "adjusted" by another matrix arising from the labeled data. Hence, our framework provides an intuitive and easy way to learn hash functions in a semisupervised paradigm.

\subsection{Nonorthogonal Projection Learning}

In the previous section, we imposed orthogonality constraints on the projection directions in order to approximately decorrelate the hash bits. However, these orthogonality constraints sometimes lead to a practical problem. It is well known that for most real-world datasets, most of the variance is contained in the top few projections. The orthogonality constraints force one to progressively pick those directions that have very low variance, substantially reducing the quality of lower bits, and hence the whole embedding. We empirically verify this behavior in Section 5. Depending on the application, it may make sense to pick a direction that is not necessarily orthogonal to the previous directions but has high variance as well as low empirical error on the labeled set. On the other hand, one doesn't want to pick a previous direction again since the fixed thresholds will generate the same hash codes in our case. Hence, instead of imposing hard orthogonality constraints, we convert them into a penalty term added to the objective function. This allows the learning algorithm to pick suitable directions by balancing various terms. With this, one can write the new objective function as

$$
\begin{aligned}
J(\mathbf{W}) & =\frac{1}{2} \operatorname{tr}\left\{\mathbf{W}^{\top} \mathbf{M W}\right\}-\frac{\rho}{2}\left\|\mathbf{W}^{\top} \mathbf{W}-\mathbf{I}\right\|_{\mathcal{F}}^{2} \\
& =\frac{1}{2} \operatorname{tr}\left\{\mathbf{W}^{\top} \mathbf{M W}\right\}-\frac{\rho}{2} \operatorname{tr}\left[\left(\mathbf{W}^{\top} \mathbf{W}-\mathbf{I}\right)^{\top}\left(\mathbf{W}^{\top} \mathbf{W}-\mathbf{I}\right)\right] .
\end{aligned}
$$

The new formulation has certain tolerance to nonorthogonality, which is modulated by a positive coefficient $\rho$. However, the above objective function is nonconvex and there is no easy way to find the global solution unlike the previous case. To maximize the objective function $J$ with respect to $\mathbf{W}$, we set the derivative to zero and absorb all constants into $\rho$ as

$$
\frac{\partial J(\mathbf{W})}{\partial \mathbf{W}}=0 \Rightarrow\left(\mathbf{W W}^{\top}-\mathbf{I}-\frac{1}{\rho} \mathbf{M}\right) \mathbf{W}=0 .
$$

Though the above equation admits infinite number of solutions, since $\mathbf{W}$ has a nonempty nullspace we can obtain a solution by ensuring

$$
\mathbf{W} \mathbf{W}^{\top} \mathbf{W}=\left(\mathbf{I}+\frac{1}{\rho} \mathbf{M}\right) \mathbf{W} .
$$


One can get a simple solution for the above condition if $\mathbf{I}+$ $\frac{1}{\rho} \mathbf{M}$ is positive definite. From (21), $\mathbf{M}$ is symmetric but not necessarily positive definite. Let $\mathbf{Q}=\mathbf{I}+\frac{1}{\rho} \mathbf{M}$. Clearly, $\mathbf{Q}$ is also symmetric. In the following proposition, we show that $\mathbf{Q}$ is positive definite if the coefficient $\rho$ is chosen appropriately.

Proposition 4.1. The matrix $\mathbf{Q}$ is positive definite if $\rho>$ $\max \left(0,-\bar{\lambda}_{\text {min }}\right)$, where $\bar{\lambda}_{\text {min }}$ is the smallest eigenvalue of $\mathbf{M}$.

Proof. By definition in (25), $\rho>0$. Since $\mathbf{M}$ is symmetric, it can be represented as $\mathbf{M}=\mathbf{U} \operatorname{diag}\left(\lambda_{1}, \ldots, \lambda_{D}\right) \mathbf{U}^{\top}$, where all $\lambda_{i} \mathrm{~s}$ are real. Let $\bar{\lambda}_{\text {min }}=\min \left(\lambda_{1}, \ldots, \lambda_{D}\right)$. Then, $\mathbf{Q}$ can be written as

$$
\begin{aligned}
\mathbf{Q} & =\mathbf{I}+\mathbf{U} \operatorname{diag}\left(\frac{\lambda_{1}}{\rho}, \ldots, \frac{\lambda_{D}}{\rho}\right) \mathbf{U}^{\top} \\
& =\mathbf{U} \operatorname{diag}\left(\frac{\lambda_{1}}{\rho}+1, \ldots, \frac{\lambda_{D}}{\rho}+1\right) \mathbf{U}^{\top} .
\end{aligned}
$$

Clearly, $\mathbf{Q}$ will have all eigenvalues positive if $\frac{\lambda_{\min }}{\rho}+1>$ $0 \Rightarrow \rho>-\lambda_{\min }$.

If $\mathbf{Q}$ is positive definite, it can be decomposed as $\mathbf{Q}=$ $\mathbf{L L}^{\top}$ using Cholesky decomposition. Then, one can easily verify that $\mathbf{W}=\mathbf{L U}$ satisfies (27). To achieve a meaningful approximate solution to our problem, we truncate the computed matrix $\mathbf{W}$ by selecting its first $k$ columns. The final nonorthogonal projections are derived as

$$
\mathbf{W}_{\text {nonorth }}=\mathbf{L U}_{k},
$$

where $\mathbf{U}_{k}$ are the top $k$ eigenvectors of $\mathbf{M}$.

\subsection{Sequential Projection Learning}

The above nonorthogonal solution is achieved by adjusting the previous orthogonal solution in a single step. However, this is one of many possible solutions which tend to work well in practice. One potential issue is that the above nonorthogonal solution is sensitive to the choice of the penalty coefficient $\rho$. To address these concerns, we further propose an alternative solution to learn a sequence of projections which implicitly incorporates bit correlation by iteratively updating the pairwise label matrix. In addition, this iterative solution has the sequential error correction property where each hash function tries to correct the errors made by the previous one.

The idea of sequential projection learning is quite intuitive. The hash functions are learned iteratively such that at each iteration, the pairwise label matrix $\mathbf{S}$ in (14) is updated by imposing higher weights on point pairs violated by the previous hash function. This sequential process implicitly creates dependency between bits and progressively minimizes empirical error. The sign of $\mathbf{S}_{i j}$, representing the logical relationship in a point pair $\left(\mathbf{x}_{i}, \mathbf{x}_{j}\right)$, remains unchanged in the entire process and only its magnitude $\left|\mathbf{S}_{i j}\right|$ is updated. Algorithm 1 describes the procedure of the proposed semisupervised sequential projection learning method.

Algorithm 1. Sequential projection learning for hashing $(S P L H)$

Input: data $\mathbf{X}$, pairwise labeled data $\mathbf{X}_{l}$, initial pairwise labels $\mathbf{S}_{1}$, length of hash codes $K$, constant $\alpha$

for $k=1$ to $K$ do

Compute adjusted covariance matrix:

$$
\mathbf{M}_{k}=\mathbf{X}_{l} \mathbf{S}_{k} \mathbf{X}_{l}^{\top}+\eta \mathbf{X} \mathbf{X}^{\top}
$$

Extract the first eigenvector $\mathbf{e}$ of $\mathbf{M}_{k}$ and set: $\mathbf{w}_{k}=\mathbf{e}$

Update the labels from vector $\mathbf{w}_{k}$ :

$$
\mathbf{S}_{k+1}=\mathbf{S}_{k}-\alpha \mathrm{T}\left(\tilde{\mathbf{S}}^{k}, \mathbf{S}_{k}\right)
$$

Compute the residual:

$$
\mathbf{X}=\mathbf{X}-\mathbf{w}_{k} \mathbf{w}_{k}^{\top} \mathbf{X}
$$

end for

Suppose $\tilde{\mathbf{S}}^{k} \in \mathbb{R}^{l \times l}$ measures the signed magnitude of pairwise relationships of the $k$ th projections of $\mathbf{X}_{l}$ :

$$
\tilde{\mathbf{S}}^{k}=\mathbf{X}_{l}^{\top} \mathbf{w}_{k} \mathbf{w}_{k}^{\top} \mathbf{X}_{l} .
$$

Mathematically, $\tilde{\mathbf{S}}^{k}$ is simply the derivative of empirical accuracy of the $k$ th hash function, i.e., $\tilde{\mathbf{S}}^{k}=\nabla_{\mathbf{S}} J_{k}$, where $J_{k}=\mathbf{w}_{k}^{\top} \mathbf{X}_{l} \mathbf{S} \mathbf{X}_{l}^{\top} \mathbf{w}_{k}$. The function $\mathrm{T}(\cdot)$ implies the truncated gradient of $J_{k}$ :

$$
\mathrm{T}\left(\tilde{\mathbf{S}}_{i j}^{k}, \mathbf{S}_{i j}\right)=\left\{\begin{aligned}
\tilde{\mathbf{S}}_{i j}^{k}: & \operatorname{sgn}\left(\mathbf{S}_{i j} \cdot \tilde{\mathbf{S}}_{i j}^{k}\right)<0 \\
0: & \operatorname{sgn}\left(\mathbf{S}_{i j} \cdot \tilde{\mathbf{S}}_{i j}^{k}\right) \geq 0 .
\end{aligned}\right.
$$

The condition $\operatorname{sgn}\left(\mathbf{S}_{i j} \cdot \tilde{\mathbf{S}}_{i j}^{k}\right)<0$ for a labeled pair $\left(\mathbf{x}_{i}, \mathbf{x}_{j}\right)$ indicates that hash bits $h_{k}\left(\mathbf{x}_{i}\right)$ and $h_{k}\left(\mathbf{x}_{j}\right)$ contradict the given pairwise label. In other words, points in a neighbor pair $\left(\mathbf{x}_{i}, \mathbf{x}_{j}\right) \in \mathcal{M}$ are assigned different bits or those in $\left(\mathbf{x}_{i}, \mathbf{x}_{j}\right) \in \mathcal{C}$ are assigned the same bit. For each such violation, $\mathbf{S}_{i j}$ is updated as $\mathbf{S}_{i j}=\mathbf{S}_{i j}-\alpha \tilde{\mathbf{S}}_{i j}^{k}$. The step size $\alpha$ is chosen such that $\alpha \leq \frac{1}{\beta}$, where $\beta=\max _{i}\left\|\mathbf{x}_{i}\right\|^{2}$, ensuring $\left|\alpha \tilde{\mathbf{S}}_{i j}^{k}\right| \leq 1$. This leads to numerically stable updates without changing the sign of $\mathbf{S}_{i j}$. Those pairs for which current hash function produces the correct bits, i.e., $\operatorname{sgn}\left(\mathbf{S}_{i j} \cdot \tilde{\mathbf{S}}_{i j}^{k}\right)>0, \mathbf{S}_{i j}$ is kept unchanged by setting $\mathrm{T}\left(\tilde{\mathbf{S}}_{i j}^{k}, \mathbf{S}_{i j}\right)=0$. Thus those labeled pairs for which the current hash function does not predict the bits correctly exert more influence on the learning of the next function, biasing the new projection to produce correct bits for such pairs. Intuitively, it has a flavor of boosting-based methods commonly used for classification. However, unlike the standard boosting framework used for supervised learning, such as the one used in [18], the proposed method performs the sequential learning in a semi-supervised scenario, where the objective is to maximize the accuracy of the binary partition while maintaining the balancing of the partition. Furthermore, during the indexing and search process, the derived hash functions are treated equally instead of being weighted, as in the boosting method.

After extracting a projection direction using $\mathbf{M}_{k}$, the contribution of the subspace spanned by that direction is removed from $\mathbf{X}$ to minimize the redundancy in bits. Note that this is not the same as imposing the orthogonality constraints on $\mathbf{W}$ discussed earlier. Since the supervised term $\mathbf{X}_{l} \mathbf{S}_{k} \mathbf{X}_{l}^{\top}$ still contains information potentially from the whole space spanned by original $\mathbf{X}$, the new direction may still have a component in the subspace spanned by the previous directions. Thus, the proposed formulation automatically decides the level of desired correlations between successive hash functions. If empirical accuracy is not affected, it prefers to pick uncorrelated projections. Unlike the nonorthogonal solution discussed in Section 4.2, the proposed sequential method aggregates various desirable properties in a single formulation leading to superior performance on real-world tasks as shown in Section 5. In 


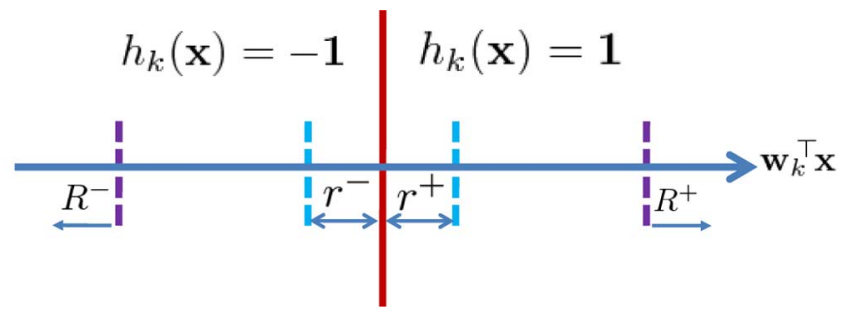

Fig. 2. Potential errors due to thresholding (red line) of the projected data to generate a bit. Points in $r^{-}$and $r^{+}$are assigned different bits even though they are quite close. Also, points in $R^{-}\left(R^{+}\right)$and $r^{-}\left(r^{+}\right)$are assigned the same bit even though they are quite far.

fact, one can extend this sequential learning method in unsupervised cases as well, as shown in the next section.

\subsection{Unsupervised Sequential Projection Learning}

Unlike the semi-supervised case, pairwise labels are not available in the unsupervised case. To apply the general framework of sequential projection learning to an unsupervised setting, we propose the idea of generating pseudolabels at each iteration of learning. While generating a bit via a binary hash function, there are two types of boundary errors one encounters due to thresholding of the projected data. Suppose all the data points are projected on a 1D axis, as shown in Fig. 2, and the red vertical line is the partition boundary, i.e., $\mathbf{w}_{k}^{\top} \mathbf{x}=0$. The points left to the boundary are assigned a hash value $h_{k}(\mathbf{x})=-1$ and those on the right are assigned a value $h_{k}(\mathbf{x})=1$. The regions marked as $r^{-}, r^{+}$are located very close to the boundary and regions $R^{-}, R^{+}$are located far from it. Due to thresholding, points in the pair $\left(\mathbf{x}_{i}, \mathbf{x}_{j}\right)$, where $\mathbf{x}_{i} \in r^{-}$and $\mathbf{x}_{j} \in r^{+}$, are assigned different hash bits even though their projections are quite close. On the other hand, points in pair $\left(\mathbf{x}_{i}, \mathbf{x}_{j}\right)$, where $\mathbf{x}_{i} \in r^{-}$and $\mathbf{x}_{j} \in R^{-}$or $\mathbf{x}_{i} \in r^{+}$and $\mathbf{x}_{i} \in R^{+}$, are assigned the same hash bit even though their projected values are quite far apart. To correct these two types of boundary "errors," we first introduce a neighbor-pair set $\mathcal{M}$ and a nonneighbor-pair set $\mathcal{C}$ :

$$
\begin{aligned}
& \mathcal{M}=\left\{\left(x_{i}, x_{j}\right)\right\}: h\left(x_{i}\right) \cdot h\left(x_{j}\right)=-1,\left|\mathbf{w}^{\top}\left(\mathbf{x}_{i}-\mathbf{x}_{j}\right)\right| \leq \epsilon, \\
& \mathcal{C}=\left\{\left(x_{i}, x_{j}\right)\right\}: h\left(x_{i}\right) \cdot h\left(x_{j}\right)=1,\left|\mathbf{w}^{\top}\left(\mathbf{x}_{i}-\mathbf{x}_{j}\right)\right| \geq \zeta .
\end{aligned}
$$

Then, given the current hash function, a desired number of point pairs are sampled from both $\mathcal{M}$ and $\mathcal{C}$. Suppose $\mathbf{X}_{\mathcal{M C}}$ contains all the points that are part of at least one sampled pair. Using the labeled pairs and $\mathbf{X}_{\mathcal{M C}}$, a pairwise label matrix $\mathbf{S}_{\mathcal{M C}}^{k}$ is constructed similar to (14). In other words, for a pair of samples $\left(\mathbf{x}_{i}, \mathbf{x}_{j}\right) \in \mathcal{M}$, a pseudolabel $\mathbf{S}_{\mathcal{M C}}^{k}=1$ is assigned while for those $\left(\mathbf{x}_{i}, \mathbf{x}_{j}\right) \in \mathcal{C}, \mathbf{S}_{\mathcal{M C}}^{k}=-1$ is assigned. In the next iteration, these pseudolabels enforce a point pair in $\mathcal{M}$ to be assigned the same hash values and those in $\mathcal{C}$ different ones. Thus, it sequentially tries to correct the potential errors made by the previous hash functions. Note that the above discussion is based on the assumption that the pairs sampled from the close regions of opposite sides of the boundary are potential neighbors. In general, when the splitting hyperplane passes through the dense regions of data distribution, this assumption will be met. But when the hyperplane passes through sparse regions, it may be violated. Moreover, the number of available pseudopairs may be too small to learn the next hash function reliably.

Algorithm 2. Unsupervised sequential projection learning for hashing (USPLH)

Input: data $\mathbf{X}$, length of hashing codes $K$

Initialize $\mathbf{X}_{\mathcal{M C}}^{0}=\emptyset, \mathbf{S}_{\mathcal{M C}}^{0}=\mathbf{0}$.

for $k=1$ to $K$ do

Compute adjusted covariance matrix:

$$
\mathbf{M}_{k}=\sum_{i=0}^{k-1} \delta^{k-i} \mathbf{X}_{\mathcal{M C}}^{i} \mathbf{S}_{\mathcal{M C}}^{i} \mathbf{X}_{\mathcal{M C}}^{i}+\eta \mathbf{X} \mathbf{X}^{\top}
$$

Extract the first eigenvector e of $\mathbf{M}_{k}$ and set:

$$
\mathbf{w}_{k}=\mathbf{e}
$$

Generate pseudo labels from projection $\mathbf{w}_{k}$ : Sample $\mathbf{X}_{\mathcal{M C}}^{k}$ and construct $\mathbf{S}_{\mathcal{M C}}^{k}$

Compute the residual:

\section{end for}

$$
\mathbf{X}=\mathbf{X}-\mathbf{w}_{k} \mathbf{w}_{k}^{\top} \mathbf{X}
$$

Note that each hash function $h_{k}(\cdot)$ produces a pseudolabel set $\mathbf{X}_{\mathcal{M C}}^{k}$ and the corresponding label matrix $\mathbf{S}_{\mathcal{M C}}^{k}$. The new label information is used to adjust the data covariance matrix in each iteration of sequential learning, similar to that for the semi-supervised case. However, the unsupervised setting does not have a boosting-like update of the label matrix, unlike the semi-supervised case. Each iteration results in its own pseudolabel matrix, depending on the hash function. Hence, to learn a new projection, all the pairwise label matrices since the beginning are used but their contribution is decayed exponentially by a factor $\delta$ at each iteration. Note that one does not need to store these matrices explicitly since incremental update can be done at each iteration, resulting in the same memory and time complexity as for the semisupervised case. The detailed learning procedure is described in Algorithm 2. Since there exist no pseudolabels at the beginning, the first vector $\mathbf{w}_{1}$ is just the first principal direction of the data. Then, each hash function is learned to satisfy the pseudolabels iteratively by adjusting the data covariance matrix, similarly to the SPLH approach.

To summarize, besides the three different versions of semi-supervised hashing methods, i.e., the orthogonal solution $S S H_{\text {orth }}$, the nonorthogonal solution $S S H_{\text {nonorth }}$ and the sequential solution SPLH, we also proposed an unsupervised extension of the sequential learning method in this section, named USPLH.

\section{EXPERIMENTS}

We evaluated all three versions of the proposed semisupervised hashing methods, including orthogonal solution $S S H_{\text {orth }}$, nonorthogonal solution $S S H_{\text {nonorth }}$ (with orthogonality constraint relaxed), and sequential solution $S P L H$, as well as the unsupervised extension (USPLH), on several benchmark datasets. Their performance is compared with other popular binary coding methods, including Locality Sensitive Hashing, Spectral Hashing, Binary Reconstructive Embedding, Boosted Similarity Sensitive Coding, and Shift Invariant Kernel-based Hashing (SIKH). These methods cover both unsupervised and supervised categories. Previous works have shown that $\mathrm{SH}$ performs better than other binary encoding methods [13], such as Restricted Boltzmann 
Machines (RBMs) [19] and BSSC [18]. For both SH and BRE, we used the best setting reported in previous literature. For $L S H$, we randomly select projections from a Gaussian distribution with zero-mean and identity covariance and apply random partitioning to construct hash functions. In addition, for all the supervised and semi-supervised methods, a small set of labeled samples was used during training. For example, only 1,000 labeled images are used in the experiments on the CIFAR10 dataset and 2,000 on Flickr image data. Finally, for the proposed approach, we used cross validation to determine some parameters such as the weight coefficient $\eta$ and the step size $\alpha$.

In the following sections, we first discuss our evaluation protocols, followed by brief description of the benchmark datasets. Finally, extensive experimental results and comparisons are presented.

\subsection{Evaluation Protocols}

To perform fair evaluation, we adopt two criteria commonly used in the literature:

1. Hamming ranking. All the points in the database are ranked according to their Hamming distance from the query and the desired neighbors are returned from the top of the ranked list. The complexity of Hamming ranking is linear even though it is very fast in practice.

2. Hash lookup. A lookup table is constructed using the database codes and all the points in the buckets that fall within a small Hamming radius $r$ of the query are returned. The complexity of the hash lookups is constant time.

Note that evaluations based on Hamming ranking and hash lookup focus on different characteristics of hashing techniques. For instance, hash lookup emphasizes the practical search speed more. However, when using many hash bits and a single hash table, the Hamming space becomes increasingly sparse, and very few samples fall within the Hamming radius $r(r=2$ in our setting), resulting in many failed queries without returned data points. In this situation, Hamming ranking provides better quality measurement of the Hamming embedding while neglecting the issue of the search speed. All the experiments were conducted using a single hash table with relatively compact codes (up to 64 bits for the largest image collection dataset with around 80 million points). The search results are evaluated based on whether the returned images and the query sample share the same semantic labels for supervised and semi-supervised tests. We use several metrics to measure the quantitative performance of different methods. For Hamming ranking-based evaluation, we compute the retrieval precision as the percentage of true neighbors among the top $M$ returned samples, where $M$ is uniformly set as 500 in the experiments. Finally, similarly to [13], a Hamming radius of 2 is used to retrieve the neighbors in the case of hash lookup. The precision of the returned samples falling within Hamming radius 2 is reported. If a query returns no neighbors inside the Hamming ball with radius 2, it is treated as a failed query with zero precision.

\subsection{Data Sets}

We used three image datasets in our experiments, i.e., CIFAR-10, a Flickr image collection, and the 80 million tiny images, with the number of samples ranging from tens

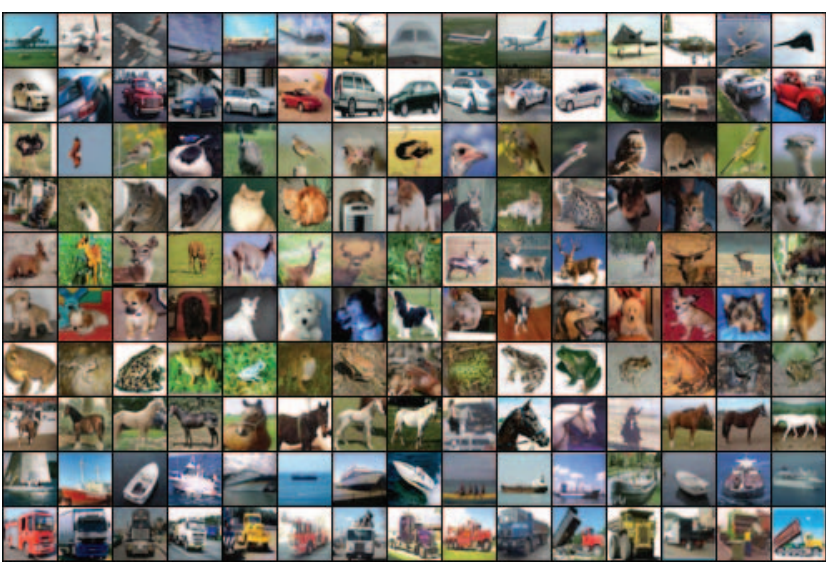

Fig. 3. A few example images from the CIFAR10 dataset. From top row to bottom row, the image classes are airplane, automobile, bird, cat, deer, dog, frog, horse, ship, and truck.

of thousands to millions. In addition, we use 1 million SIFT feature vectors for the experiments with unsupervised hashing methods. For the first two datasets, since they are fully annotated, we focus on the quantitative evaluation of the search accuracy. For the 80 million image data, we demonstrate the scalability of the proposed methods and provide qualitative evaluation by providing the search results of some exemplar queries.

\subsubsection{CIFAR-10 Data Set}

The CIFAR-10 dataset is a labeled subset of the 80-million tiny images collection [31]. It consists of a total of 60,000 $32 \times 32$ color images in 10 classes, each of which has 6,000 samples. ${ }^{1}$ Each sample in this dataset is associated with a mutually exclusive class label. A few example images from the CIFAR10 dataset are shown in Fig. 3. Since this dataset is fully annotated, the ground truth semantic neighbors can be easily retrieved based on the class labels. The entire dataset is partitioned into two parts: a training set with 59,000 samples and a test set with 1,000 samples. The training set is used for learning hash functions and constructing the hash lookup tables. For BSSC, BRE, and the proposed semi-supervised hashing methods, we additionally sample 1,000 random points from the training set and assign semantic nearest neighbor information (i.e., construct the pairwise label matrix $\mathbf{S}$ ) based on the image labels. The binary encoding is performed in the 384dim GIST feature space [32].

\subsubsection{Flickr Images}

Here, we use a set of Flickr consumer images collected by the NUS lab, i.e., the NUS-WIDE dataset [33]. It was created as a benchmark for evaluating multimedia search techniques. This dataset contains around 270,000 images associated with 81 ground truth concept tags. Unlike the CIFAR10 dataset, each sample in NUS-WIDE could be assigned multiple labels since this kind of multiple tagging occurs very often in a realworld annotation scenario. Compared to the CIFAR-10 dataset, the NUS-WIDE dataset contains images with much higher resolutions, which allow us to use a Bag-of-VisualWord model with local SIFT features for extracting the image

1. http://www.cs.toronto.edu/ kriz/cifar.html. 


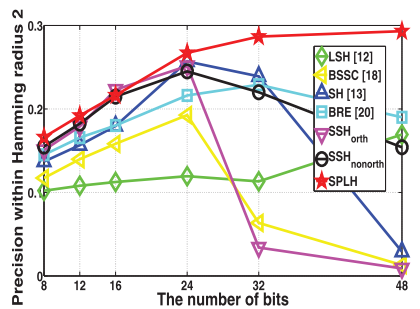

(a)

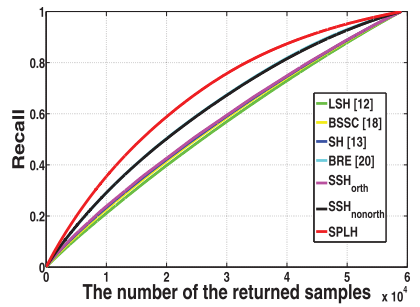

(c)

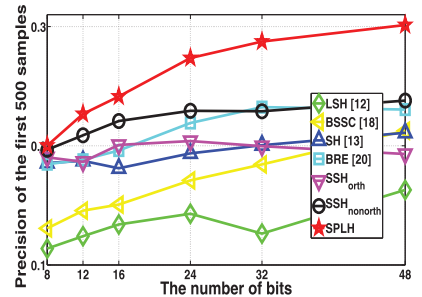

(b)

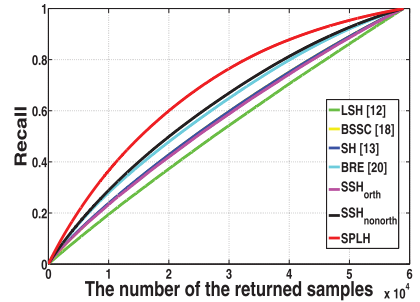

(d)

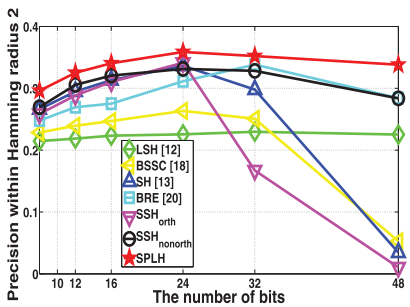

(a)

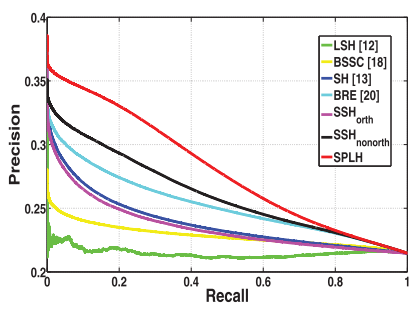

(c)

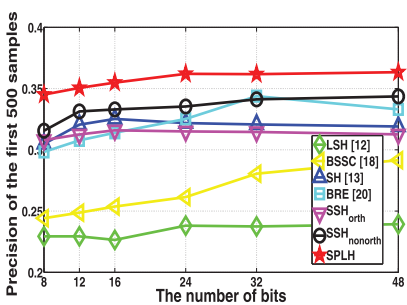

(b)

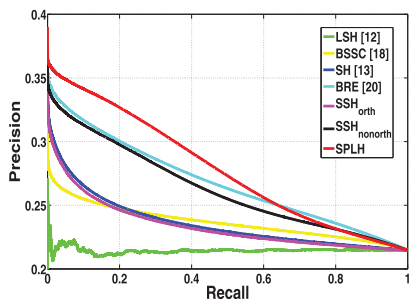

(d)
Fig. 4. Results on the CIFAR10 dataset. (a) Precision within Hamming radius 2 using hash lookup. (b) Precision of the top 500 returned samples using Hamming ranking. (c) Recall curves with 24 bits. (d) Recall curves with 32 bits. LSH: Locality Sensitive Hashing, BSSC: Boosted Similarity Sensitive Coding, SH: Spectral Hashing, BRE: Binary Reconstructive Embedding, $\mathrm{SSH}_{\text {orth }}$ : Orthogonal Semi-Supervised Hashing, $S S H_{\text {nonorth }}$ : Nonorthogonal Semi-Supervised Hashing, and SPLH: Sequential Projection Learning-based Hashing.

descriptor [34]. Particularly, a visual vocabulary with 500length code book and a soft assignment strategy was used for deriving the image features, as described in [35].

Similarly to the CIFAR10 dataset, we partitioned this set into two parts, $1 \mathrm{~K}$ for query test and around $269 \mathrm{~K}$ for training and constructing hash tables. In addition, $2 K$ images are randomly sampled with labels for $B S S C, B R E$, and our semisupervised hashing methods. The precision is evaluated based on whether the returned images and the query share at least one common semantic label. The performance was evaluated with different code lengths varying from 8-bit to 64-bit.

\subsubsection{SIFT-1M Data Set}

We also test the performance of our unsupervised sequential learning method (USPLH) using the SIFT-1M dataset. It contains 1 million local SIFT descriptors extracted from a large set of images described in [27]. Each point in the dataset is a 128-dim vector representing histograms of gradient orientations. We use 1 million samples for training and an additional $10 K$ for testing. Euclidean distance is used to determine the nearest neighbors. Following the criterion used in [36], [13], a returned point is considered a good neighbor if it lies in the top 2 percentile points closest to a query. Since no labels are available in this experiment, both $S S H_{\text {orth }}$ and $S S H_{\text {nonorth }}$ have no adjustment term. Because it results in the same hash functions by using just principal projections, we named it PCA-based hashing $(P C A H)$. We also compared with a few unsupervised hashing techniques, including $L S H, S H$, and SIKH on this dataset. For USPLH, to learn each hash function sequentially, we select 2,000 samples from each of the four boundary and margin regions $r^{-}, r^{+}, R^{-}, R^{+}$. A label matrix $\mathbf{S}$ is constructed by assigning pseudolabels to pairs generated from these samples.
Fig. 5. Results on the Flickr image dataset. (a) Precision within Hamming radius 2 using hash lookup. (b) Precision of the top 500 returned samples using Hamming ranking. (c) Precision-Recall curve with 24 bits. (d) Precision-Recall curve with 32 bits. LSH: Locality Sensitive Hashing, BSSC: Boosted Similarity Sensitive Coding, SH: Spectral Hashing, BRE: Binary Reconstructive Embedding, $\mathrm{SSH}_{\text {orth }}$ : Orthogonal Semi-Supervised Hashing, $S S H_{\text {nonorth }}$ : Nonorthogonal Semi-Supervised Hashing, and SPLH: Sequential Projection Learningbased Hashing.

\subsubsection{Eighty Million Tiny Images}

Besides the quantitative evaluation on the above three datasets, we also apply our techniques on a large collection of images with Gist features, i.e., 80 million tiny images dataset [31], which has been used as a benchmark dataset for designing binary encoding approaches [13], [20], [37], [22]. However, only a small portion of the dataset is manually labeled and the associated meta-information is fairly noisy. Since CIFAR10 is a fully annotated subset of this gigantic image collection, we combine these two datasets in our experiments. The experimental protocol for evaluation is described as below. A subset of two million data points is sampled to construct the training set, especially for computing the data covariance matrix for all the eigendecomposition-based approaches, and a separate set of $2 \mathrm{~K}$ samples from the CIFAR10 dataset is used as labeled samples. For $\mathrm{SH}$, the hash functions were designed using this two-million dataset. For BRE and the proposed semisupervised hashing methods, both the two-million dataset and $2 \mathrm{~K}$ labeled data were used to learn the hash functions. After obtaining the hash functions, the Hamming embedding of the entire dataset with a total of 79,302,017 samples is computed with 64-bit hash codes. Finally, some examples are randomly selected for query test, and qualitative comparison is made with other methods.

\subsection{Results}

For quantitative evaluation on the CIFAR10 and Flickr datasets, the number of bits is varied from 8 to 48 for the CIFAR10 dataset and the Flickr image dataset. The performance curves are shown in Figs. 4 and 5, respectively, where the precision curves of both Hamming ranking and hash lookup (within Hamming radius 2) show the accuracy of the returned top results. From these figures, it is not very 


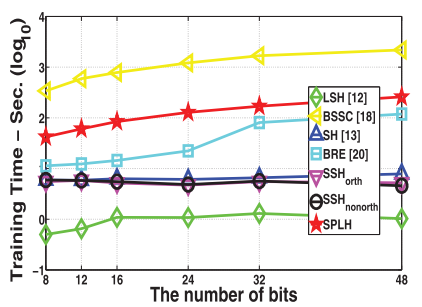

(a)

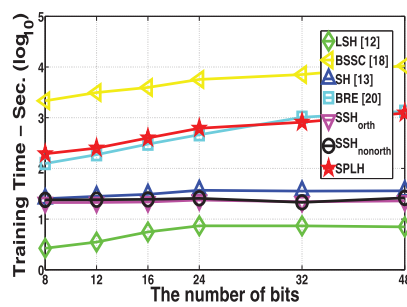

(c)

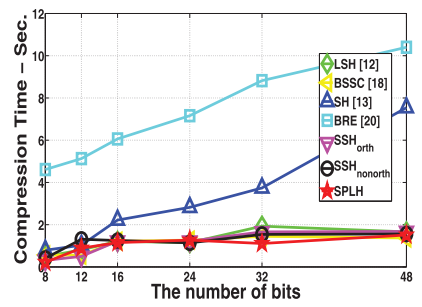

(b)

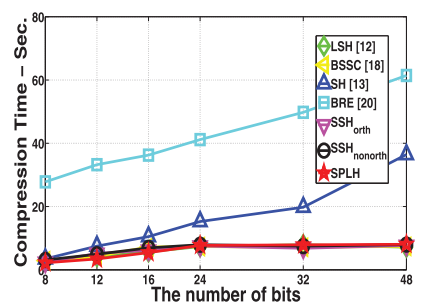

(d)
Fig. 6. Computational cost for different binary encoding methods. (a) Training cost on the CIFAR10 dataset. (b) Compression cost on the CIFAR10 dataset. (c) Training cost on the Flickr dataset. (d) Compression cost on the Flickr dataset. LSH: Locality Sensitive Hashing, BSSC: Boosted Similarity Sensitive Coding, SH: Spectral Hashing, BRE: Binary Reconstructive Embedding, $S S H_{\text {orth }}$ : Orthogonal Semi-Supervised

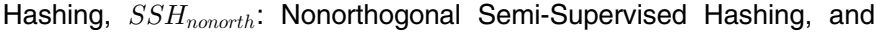
SPLH: Sequential Projection Learning-based Hashing.

surprising to see that $L S H$ provides the worst performance since the random hash functions lack discrimination for small bit lengths. The orthogonal solution for $\mathrm{SSH}$ described in Section 4, i.e., $S S H_{\text {orth }}$, has comparable performance to the nonorthogonal solution, $S S H_{\text {nonorth }}$, for a small number of bits (i.e., 8,12 , and 16 bits) since there is enough variance in the top few orthogonal directions computed in our semisupervised formulation. But when using a large number of bits, $S S H_{\text {orth }}$ performs much worse since the orthogonal solution forces one to progressively pick the low variance projections, substantially reducing the quality of the whole embedding. Both Figs. 4 and 5 clearly show that $S S H_{\text {nonorth }}$ is significantly better than $S S H_{\text {orth }}$ when using long hash codes. Note that although $\mathrm{SH}$ also uses principal projections directions, it can somewhat alleviate the negative impact of low variance projections by reusing the large variance projections with higher frequency sinusoidal binarization. The sequential method of $S S H$, i.e., SPLH, provides the best performance for all bits. Particularly, in the evaluation of hash lookup within Hamming radius 2 (Figs. 4a and 5a), the precision for most of the compared methods drops significantly when longer codes are used. This is because, for longer codes, the number of points falling in a bucket decreases exponentially. Thus, many queries fail by not returning any neighbor even in a Hamming ball of radius 2 . This shows a practical problem with hash lookup tables even though they have faster query response than Hamming ranking. Even in this case, SPLH provides the best performance for most of the cases. Also, the drop in precision for longer codes is much less compared to others, indicating fewer failed queries for SPLH.

As a complementary evaluation, the recall curves for CIFAR10 set are given in Figs. $4 \mathrm{c}$ and $4 \mathrm{~d}$ and the precisionrecall curves for Flickr set are given in Figs. 5c and 5d. The results demonstrate significant performance improvement

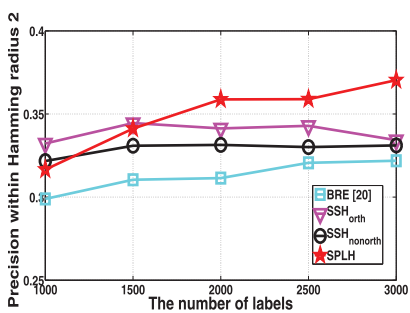

(a)

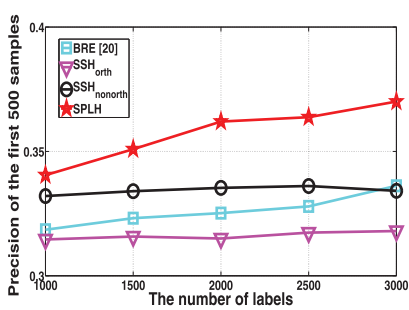

(b)
Fig. 7. Evaluation of the performance using different amounts of training samples. (a) Precision within Hamming radius 2 using hash lookup on the Flickr dataset. (b) Precision of the top 500 returned samples using Hamming ranking on the Flickr dataset. BRE: Binary Reconstructive Embedding, $S S H_{\text {orth }}$ : Orthogonal Semi-Supervised Hashing, $S S H_{\text {nonorth }}$ : Nonorthogonal Semi-Supervised Hashing, and SPLH: Sequential Projection Learning-based Hashing.

using the proposed semi-supervised hashing approaches, especially SPLH, over other methods. Differently from the CIFAR10 dataset, the Flickr dataset contains images with multiple labels. Therefore, the performance measure solely based on recall is incomplete for this multilabel problem [38]. Instead, average precision, approximated by the area under the precision-recall curves, is a more appropriate measure.

We implemented the proposed methods and other hashing approaches in Matlab and ran the experiments on a Lenovo workstation with $3.16 \mathrm{GHz}$ Quad Core CPU. Fig. 6 reports the comparison of the computational cost, including training time and compression time, for different techniques. The training time indicates the cost of learning the hash functions from training data and the compression time measures the encoding time from the original test data to binary codes. It is not surprising that $\mathrm{LSH}$ needs negligible training time since the projections are randomly generated instead of being learned. The three eigenvalue decomposition-based techniques, i.e., $S H, S S H_{\text {orth }}$, and $\mathrm{SH}_{\text {nonorth }}$, incur similar training cost. Since SPLH needs to update pairwise label matrix and performs eigenvalue decomposition at each iteration, its training time is longer but comparable to $B R E$, and much less than BSSC. Compared with offline training cost, the compression time is usually more important in practice since it is done in real time. As shown in Figs. $6 \mathrm{~b}$ and $6 \mathrm{~d}, B R E$ is the most expensive method in terms of computing the binary codes. $\mathrm{SH}$ requires a little more time than the remaining methods due to the calculation of the sinusoidal function. The code generation time can be ranked as: $\mathrm{BRE} \gg \mathrm{SH}>\mathrm{LSH} \simeq$ $\mathrm{BSSC} \simeq S S H_{\text {orth }} \simeq S S H_{\text {nonorth }} \simeq \mathrm{SPLH}$.

In all the above experiments, we fixed the size of the training subset, e.g., 2,000 for the Flickr dataset. To study the impact of the amount of supervision on the search performance, we conducted further experiments on the Flickr dataset using 24-bit hash functions while varying the number of training samples from 1,000 to 3,000. Since $B R E$ achieved comparable performance, we also compared against its performance in these tests. Fig. 7 gives the precision curves for both hash lookup and Hamming ranking for the proposed semi-supervised methods and supervised $B R E$ approach. It is clear that 2,000 points were sufficient for the Flickr dataset to obtain reasonable performance for most of the approaches. Further, adding more training data increases the training cost without adding much benefit. 


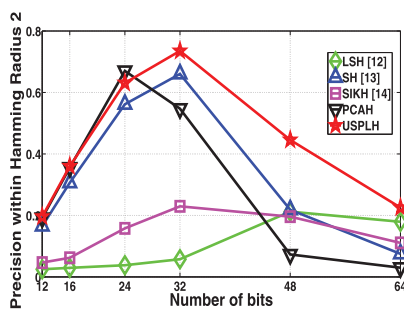

(a)

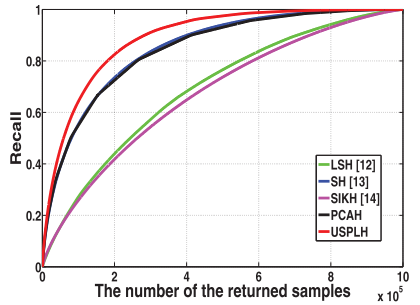

(c)

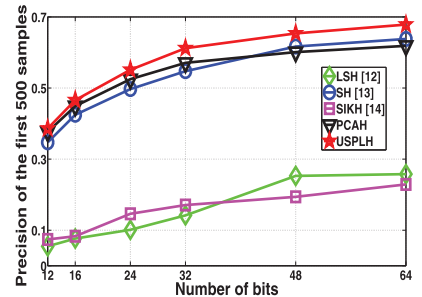

(b)

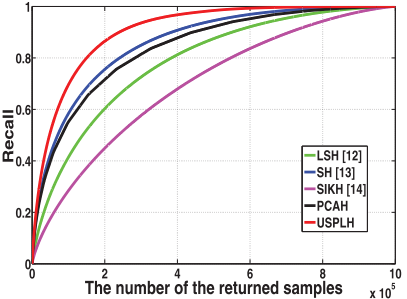

(d)
Fig. 8. Results on the SIFT-1M dataset. (a) Precision of the top 500 returned samples using Hamming ranking. (b) Precision within Hamming radius 2 using hash lookup. Recall curves (c) with 24 bits and (d) with 48 bits. LSH: Locality Sensitive Hashing, SH: Spectral Hashing, SIKH: Shift Invariant Kernel-based Hashing, and USPLH: Unsupervised Sequential Projection Learning-based Hashing.

Fig. 8 shows the experimental results of the unsupervised tests on SIFT-1M dataset. Fig. 8a shows precision curves for different methods using hash lookup table, and Fig. $8 \mathrm{~b}$ shows the precision curves using Hamming ranking. Methods that learn data-dependent projections, i.e., USPLH, $S H$, and $P C A H$, generally perform much better than $L S H$ and SIKH. SH performs better than $P C A H$ for longer codes since, for this dataset, $S H$ tends to pick the high-variance directions again. USPLH gives the best performance for most cases. Also, for Hamming radius lookup experiments, the performance of USPLH does not drop as rapidly as $\mathrm{SH}$ and PCAH with increase in bits. Thus, USPLH leads to fewre query failures in comparison to other methods. Figs. $8 \mathrm{c}$ and $8 \mathrm{~d}$ show the recall curves for different methods using 24-bit and 48-bit codes. Higher precision and recall for USPLH indicate the advantage of learning hash functions sequentially, even with noisy pseudolabels.

Finally, we present the experimental results on the large 80-million image dataset to show the scalability of the proposed semi-supervised hashing methods. We use 64-bit codes to index the 384-dim Gist descriptor, which dramatically reduces the storage of the entire dataset from hundreds of gigabytes to a few hundred megabytes. A random set of queries was sampled from the database and used for tests. Here, we compared the visual search results of the three best performing methods, i.e., $B R E, S S H_{\text {nonorth }}$, and SPLH. After obtaining the search results in hash lookup (within Hamming radius $r=2$ ), we computed the euclidian distance of the collected nearest neighbors and query images in Gist feature space and then sorted the results. The top 10 returned images for a few exemplar queries are shown in Fig. 9. SPLH presents more visually consistent search results than $B R E$ and $S S H_{\text {orth }}$. This exhaustive search usually involved only around $0.005 \sim 0.01$ percent samples from the entire 80 million set, leading to a dramatic reduction in search time.

\section{Conclusions And Future Work}

In this paper, we have proposed a semi-supervised paradigm to learn efficient hash codes by simple linear mapping which can handle semantic similarity/dissimilarity among the data. The proposed method minimizes empirical loss over the labeled data coupled with an information theoretic regularizer over both labeled and unlabeled data. A series of relaxations leads to a very simple eigen-decomposition-based solution which is extremely efficient. Based on this framework, we proposed the following family of solutions:

1. Orthogonal hash functions $\left(S S H_{\text {orth }}\right)$. By adding orthogonality as hard constraints, the hash codes can be directly obtained by conducting eigen-decomposition over an adjusted covariance matrix.

2. Nonorthogonal hash functions $\left(S S H_{\text {nonorth }}\right)$. The orthogonality constraints can be relaxed as a soft penalty term in the objective function. Then, an approximate nonorthogonal solution can be obtained through adjusting the learned orthogonal solution.

3. Sequential hash functions (SPLH). The sequential method iteratively learns new hash functions such that in each iteration a new function tends to minimize the errors made by the previous one. For this, the pairwise labels are updated by imposing higher weights on point pairs violated by the previous hash function.

4. Unsupervised sequential hash functions (USPLH). The sequential learning method was extended to the unsupervised setting, where a set of pseudolabels is generated sequentially using the probable mistakes

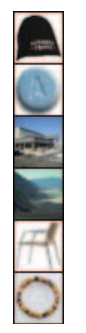

(a)

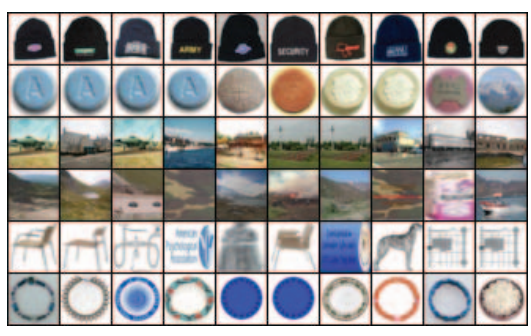

(b)

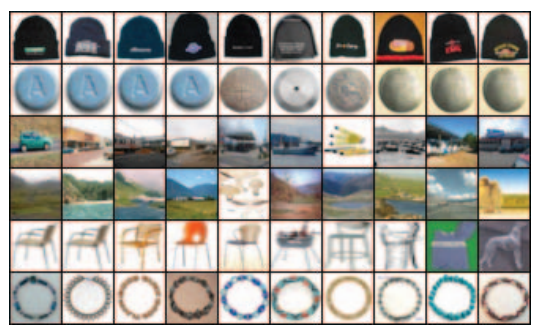

(c)

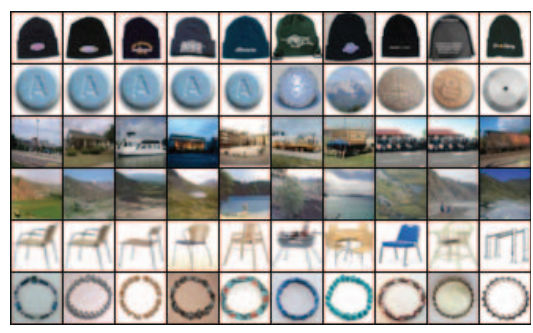

(d)

Fig. 9. Qualitative evaluation over the 80 million tiny image dataset using 64-bit codes. (a) Query images; top 10 returned images using (b) the $B R E$ method, (c) the $S S H_{\text {nonorth }}$ method, and (d) the SPLH method. BRE: Binary Reconstructive Embedding, $S S H_{\text {nonorth }}$ : Nonorthogonal SemiSupervised Hashing, and SPLH: Sequential Projection Learning-based Hashing. 
made by the previous bit. Each new hash function tries to minimize these errors subject to the same regularizer as in the semi-supervised case.

We conducted extensive experiments on four large datasets containing up to 80 million points and provided both quantitative and qualitative comparison with state-ofthe-art hashing techniques. The experimental results show superior performance of the proposed semi-supervised hashing methods. Particularly, SPLH achieved the best performance for semi-supervised and supervised cases and USPLH performs the best for unsupervised cases. The sequential techniques, i.e., SPLH and USPLH, need more time for (offline) training than $\mathrm{LSH}$ or eigen-decompositionbased methods such as $S H, S S H_{\text {orth }}$, and $S S H_{\text {nonorth }}$, but is comparable to or even faster than the BRE method, and much faster than BSSC. In terms of (online) runtime, all four proposed hashing methods are as fast as $L S H$, which is significantly faster than the $S H$ and BRE methods. In the future, we would like to investigate if any theoretical guarantees could be provided for the proposed semisupervised hashing methods.

\section{ACKNOWLEDGMENTS}

The authors thank the reviewers for their helpful comments and insights. Jun Wang was supported in part by a Google Intern Scholarship and a Chinese Government Scholarship for Outstanding Self-Financed Students Abroad. Shih-Fu Chang was supported in part by US National Science Foundation (NSF) Awards CNS-07-51078 and CNS-07-16203.

\section{REFERENCES}

[1] R. Datta, D. Joshi, J. Li, and J.Z. Wang, "Image Retrieval: Ideas, Influences, and Trends of the New Age," ACM Computing Surveys, vol. 40, no. 2, pp. 1-60, 2008.

[2] G. Shakhnarovich, T. Darrell, and P. Indyk, Nearest-Neighbor Methods in Learning and Vision: Theory and Practice. MIT Press, 2006.

[3] P. Indyk and R. Motwani, "Approximate Nearest Neighbors: Towards Removing the Curse of Dimensionality," Proc. 30th ACM Symp. Theory of Computing, pp. 604-613, 1998.

[4] J. Bentley, "Multidimensional Binary Search Trees Used for Associative Searching," Comm. ACM, vol. 18, no. 9, pp. 509-517, 1975.

[5] J.H. Friedman, J.L. Bentley, and R.A. Finkel, "An Algorithm for Finding Best Matches in Logarithmic Expected Time," ACM Trans. Math. Software, vol. 3, no. 3, pp. 209-226, 1977.

[6] C. Silpa-Anan and R. Hartley, "Optimised Kd-Trees for Fast Image Descriptor Matching," Proc. IEEE Conf. Computer Vision and Pattern Recognition, pp. 1-8, 2008.

[7] S. Omohundro, "Efficient Algorithms with Neural Network Behavior," Complex Systems, vol. 1, no. 2, pp. 273-347, 1987.

[8] J. Uhlmann, "Satisfying General Proximity/Similarity Queries with Metric Trees," Information Processing Letters, vol. 40, no. 4, pp. 175-179, 1991.

[9] P. Yianilos, "Data Structures and Algorithms for Nearest Neighbor Search in General Metric Spaces," Proc. Fourth Ann. ACM-SIAM Symp. Discrete Algorithms, pp. 311-321, 1993.

[10] M. Muja and D.G. Lowe, "Fast Approximate Nearest Neighbors with Automatic Algorithm Configuration," Proc. Int'l Conf. Computer Vision Theory and Applications, pp. 331-340, 2009.

[11] P. Indyk, "Nearest-Neighbor Searching in High Dimensions," Handbook of Discrete and Computational Geometry, J.E. Goodman and J. O'Rourke, eds., CRC Press LLC, 2004.

[12] A. Gionis, P. Indyk, and R. Motwani, "Similarity Search in High Dimensions via Hashing," Proc. 25th Int'l Conf. Very Large Data Bases, pp. 518-529, 1999.
[13] Y. Weiss, A. Torralba, and R. Fergus, "Spectral Hashing," Proc. Advances in Neural Information Processing Systems, D. Koller, D. Schuurmans, Y. Bengio, and L. Bottou, eds., vol. 21, pp. 17531760, 2008.

[14] M. Raginsky and S. Lazebnik, "Locality-Sensitive Binary Codes from Shift-Invariant Kernels," Proc. Advances in Neural Information Processing Systems 22, Y. Bengio, D. Schuurmans, J. Lafferty, C.K.I. Williams, and A. Culotta, eds., pp. 1509-1517, 2009.

[15] B. Kulis and K. Grauman, "Kernelized Locality-Sensitive Hashing for Scalable Image Search," Proc. IEEE Int'l Conf. Computer Vision, pp. 2130-2137, 2009.

[16] A. Smeulders, M. Worring, S. Santini, A. Gupta, and R. Jain, "Content-Based Image Retrieval at the End of the Early Years," IEEE Trans. Pattern Analysis and Machine Intelligence, vol. 22, no. 12, pp. 1349-1380, Dec. 2000.

[17] B. Kulis, P. Jain, and K. Grauman, "Fast Similarity Search for Learned Metrics," IEEE Trans. Pattern Analysis and Machine Intelligence, vol. 31, no. 12, pp. 2143-2157, Dec. 2009.

[18] G. Shakhnarovich, "Learning Task-Specific Similarity," PhD dissertation, Massachusetts Inst. of Technology, 2005.

[19] G. Hinton and R. Salakhutdinov, "Reducing the Dimensionality of Data with Neural Networks," Science, vol. 313, no. 5786, pp. 504507, 2006.

[20] B. Kulis and T. Darrell, "Learning to Hash with Binary Reconstructive Embeddings," Proc. Advances in Neural Information Processing Systems, Y. Bengio, D. Schuurmans, J. Lafferty, C.K.I. Williams, and A. Culotta, eds., vol. 20, pp. 1042-1050, 2009.

[21] Y. Mu, J. Shen, and S. Yan, "Weakly-Supervised Hashing in Kernel Space," Proc. IEEE Conf. Computer Vision and Pattern Recognition, pp. 3344-3351, June 2010.

[22] A. Torralba, R. Fergus, and Y. Weiss, "Small Codes and Large Image Databases for Recognition," Proc. IEEE Conf. Computer Vision and Pattern Recognition, pp. 1-8, 2008.

[23] M. Datar, N. Immorlica, P. Indyk, and V. Mirrokni, "LocalitySensitive Hashing Scheme Based on P-Stable Distributions," Proc. 12th Ann. Symp. Computational Geometry, pp. 253-262, 2004.

[24] M. Bawa, T. Condie, and P. Ganesan, "LSH Forest: Self-Tuning Indexes for Similarity Search," Proc. 14th Int'l Conf. World Wide Web, pp. 651-660, 2005.

[25] Q. Lv, W. Josephson, Z. Wang, M. Charikar, and K. Li, "MultiProbe LSH: Efficient Indexing for High-Dimensional Similarity Search," Proc. 33rd Int'l Conf. Very Large Data Bases, pp. 950-961, 2007.

[26] L. Cayton and S. Dasgupta, "A Learning Framework for Nearest Neighbor Search," Proc. Advances in Neural Information Processing Systems 20, J. Platt, D. Koller, Y. Singer, and S. Roweis, eds., pp. 233-240, 2008

[27] J. Wang, S. Kumar, and S.-F. Chang, "Sequential Projection Learning for Hashing with Compact Codes," Proc. 27th Int'l Conf. Machine Learning, J. Fürnkranz and T. Joachims, eds., pp. 11271134, June 2010.

[28] Y. Freund and R. Schapire, "A Decision-Theoretic Generalization of On-Line Learning and an Application to Boosting," Proc. Second European Conf. Computational Learning Theory, pp. 23-37, 1995.

[29] C. Fowlkes, S. Belongie, F. Chung, and J. Malik, "Spectral Grouping Using the Nystrom Method," IEEE Trans. Pattern Analysis and Machine Intelligence, vol. 26, no. 2, pp. 214-225, Feb. 2004.

[30] S. Baluja and M. Covell, "Learning to Hash: Forgiving Hash Functions and Applications," Data Mining and Knowledge Discovery, vol. 17, no. 3, pp. 402-430, 2008.

[31] A. Torralba, R. Fergus, and W. Freeman, "80 Million Tiny Images: A Large Data Set for Nonparametric Object and Scene Recognition," IEEE Trans. Pattern Analysis and Machine Intelligence, vol. 30, no. 11, pp. 1958-1970, Nov. 2008

[32] A. Oliva and A. Torralba, "Modeling the Shape of the Scene: A Holistic Representation of the Spatial Envelope," Int'l J. Computer Vision, vol. 42, no. 3, pp. 145-175, 2001.

[33] T.-S. Chua, J. Tang, R. Hong, H. Li, Z. Luo, and Y.-T. Zheng, "NusWide: A Real-World Web Image Database from National University of Singapore," Proc. ACM Conf. Image and Video Retrieval, July 2009.

[34] D.G. Lowe, "Distinctive Image Features from Scale-Invariant Keypoints," Int'l J. Computer Vision, vol. 60, no. 2, pp. 91-110, 2004

[35] Y.-G. Jiang, C.-W. Ngo, and J. Yang, "Towards Optimal Bag-ofFeatures for Object Categorization and Semantic Video Retrieval," Proc. Sixth ACM Int'l Conf. Image and Video Retrieval, pp. 494-501, 2007. 
[36] J. Wang, S. Kumar, and S.-F. Chang, "Semi-Supervised Hashing for Scalable Image Retrieval," Proc. IEEE Conf. Computer Vision and Pattern Recognition, pp. 3424-3431, June 2010.

[37] R. Fergus, Y. Weiss, and A. Torralba, "Semi-Supervised Learning in Gigantic Image Collections," Proc. Advances in Neural Information Processing Systems 22, Y. Bengio, D. Schuurmans, J. Lafferty, C.K.I. Williams, and A. Culotta, eds., pp. 522-530, 2009.

[38] R. Schapire and Y. Singer, "Boostexter: A Boosting-Based System for Text Categorization," Machine Learning, vol. 39, no. 2, pp. 135$168,2000$.

[39] W. Liu, J. Wang, S. Kumar, and S.-F. Chang, "Hashing with Graphs," Proc. 28th Int'l Conf. Machine Learning, June 2011.

[40] W. Liu, J. Wang, R. Ji, Y. Jiang, and S.-F. Chang, "Supervised Hashing with Kernels," Proc. IEEE Conf. Computer Vision and Pattern Recognition, pp. 2074-2081, June 2012.

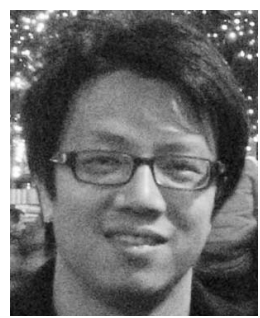

Jun Wang received the MPhil and PhD degrees from Columbia University, New York, in 2010 and 2011, respectively. Currently, he is a research staff member in the Business Analytics and Mathematical Sciences Department at the IBM T.J. Watson Research Center, Yorktown Heights, New York. He also worked as a research intern at Google New York in 2009, and as a research assistant at Harvard Medical School, Harvard University, in 2006. He has been the recipient of several awards and scholarships, including the Jury thesis award from the Department of Electrical Engineering, Columbia University, in 2011, the IBM T.J. Watson Emerging Leader in Multimedia Award 2009, the Google global intern scholarship in 2009, and a Chinese government scholarship for outstanding self-financed students abroad in 2009. His research interests include machine learning, business analytics, information retrieval, and hybrid neuralcomputer vision systems. He is a member of the IEEE.

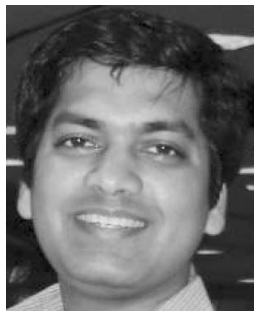

Sanjiv Kumar received the BE degree from the Birla Institute of Science and Technology, Pilani, India, and the MS degree from the Indian Institute of Technology, Chennai, India, in 1997. He received the PhD degree from The Robotics Institute, Carnegie Mellon University. From 1997 to 1999, he was a research fellow in the Department of Surgery, National University of Singapore, working in the field of medical robotics and imaging. Since 2005, he has been working at Google Research, New York, as a research scientist. His primary research interests include large-scale computer vision and machine learning, graphical models, and medical imaging. $\mathrm{He}$ is a member of the IEEE.

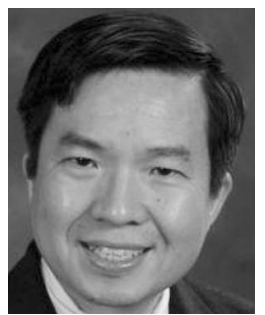

Shih-Fu Chang is the Richard Dicker Professor in the Departments of Electrical Engineering and Computer Science, and the director of the Digital Video and Multimedia Lab at Columbia University, New York. He has made significant contributions to multimedia search, visual communication, media forensics, and international standards. He has been recognized with the ACM SIGMM Technical Achievement Award, IEEE Kiyo Tomiyasu Award, Navy ONR Young Investigator Award, IBM Faculty Award, ACM Recognition of Service Award, and US National Science Foundation (NSF) CAREER Award. $\mathrm{He}$ and his students have received many Best Paper Awards, including the Most Cited Paper of the Decade Award from the Journal of Visual Communication and Image Representation. He has worked in different advising/consulting capacities for industry research labs and international institutions. He served as editor-in-chief for the IEEE Signal Processing Magazine (2006-8), and as chair of Columbia's Electrical Engineering Department (2007-2010). He is a fellow of the IEEE and a fellow of the American Association for the Advancement of Science.

For more information on this or any other computing topic, please visit our Digital Library at www.computer.org/publications/dlib. 